\title{
Detailed high-energy characteristics of AXP 4U 0142+61
}

\section{Multi-year observations with INTEGRAL, RXTE, XMM-Newton, and ASCA}

\author{
P. R. den Hartog ${ }^{1}$, L. Kuiper ${ }^{1}$, W. Hermsen ${ }^{1,2}$, V. M. Kaspi ${ }^{3}$, R. Dib ${ }^{3}$, J. Knödlseder ${ }^{4}$, and F. P. Gavriil ${ }^{5}$ \\ 1 SRON, Netherlands Institute for Space Research, Sorbonnelaan 2, 3584 CA Utrecht, The Netherlands \\ e-mail: Hartog@sron.nl \\ 2 Sterrenkundig Instituut Anton Pannekoek, University of Amsterdam, Kruislaan 403, 1098 SJ Amsterdam, The Netherlands \\ 3 Physics Department, McGill University, 3600 University Street, Montreal, PQ H3A 2T8, Canada \\ ${ }^{4}$ Centre d'Étude Spatiale des Rayonnement, CNRS/UPS, 31028 Toulouse Cedex 4, France \\ 5 NASA Goddard Space Flight Center, Astrophysics Science Division, Code 662, X-ray Astrophysics Laboratory, Greenbelt, \\ MD 20771, USA
}

Received 12 January 2008 / Accepted 3 April 2008

\section{ABSTRACT}

\begin{abstract}
4U 0142+61 is one of the Anomalous X-ray Pulsars exhibiting hard X-ray emission above $10 \mathrm{keV}$ discovered with INTEGRAL. In this paper we present detailed spectral and temporal characteristics both in the hard X-ray $(>10 \mathrm{keV})$ and soft X-ray $(<10 \mathrm{keV})$ domains obtained using data from INTEGRAL, XMM-Newton, ASCA and RXTE. Accumulating data collected over four years with the imager IBIS-ISGRI aboard INTEGRAL, the time-averaged total spectrum shows a power-law like shape with photon index $\Gamma=0.93 \pm 0.06$. $4 \mathrm{U} 0142+61$ is detected up to $229 \mathrm{keV}$ and the flux between $20 \mathrm{keV}$ and $229 \mathrm{keV}$ is $(15.01 \pm 0.82) \times 10^{-11} \mathrm{erg} \mathrm{cm}^{-2} \mathrm{~s}^{-1}$, which exceeds the energy flux in the $2-10 \mathrm{keV}$ band by a factor of $\sim 2.3$. Using simultaneously collected data with the spectrometer SPI of INTEGRAL the combined total spectrum yields the first evidence for a spectral break above $100 \mathrm{keV}$. Assuming a logparabolic function for the spectral shape above $20 \mathrm{keV}$ the peak energy of $4 \mathrm{U} 0142+61$ is $228_{-41}^{+65} \mathrm{keV}$. There is no evidence for significant long-term time variability of the total emission from $4 \mathrm{U} 0142+61$. Both the total flux and the spectral index are stable within the $17 \%$ level $(1 \sigma)$. Pulsed emission is measured with ISGRI up to $160 \mathrm{keV}$. The $20-160 \mathrm{keV}$ profile shows a broad double-peaked pulse with a $6.2 \sigma$ detection significance. The total pulsed spectrum can be described with a very hard power-law shape with a photon index $\Gamma=0.40 \pm 0.15$ and a $20-150 \mathrm{keV}$ flux of $(2.68 \pm 1.34) \times 10^{-11} \mathrm{erg} \mathrm{cm}^{-2} \mathrm{~s}^{-1}$. To perform accurate phase-resolved spectroscopy over the total X-ray window, we produced pulse profiles in absolute phase for INTEGRAL-ISGRI, RXTE-PCA, XMM-Newton-PN and ASCA-GIS. The two known pulses in all soft X-ray profiles below $10 \mathrm{keV}$ are located in the same phases. Three XMM-Newton observations in 2003-2004 show statistically identical profiles. However, we find a significant profile morphology change between an ASCA-GIS observation in 1999 following a possible glitch of 4U 0142+61. This change can be accounted for by differences in relative strengths and spectral shapes $(0.8-10 \mathrm{keV})$ of the two pulses. The principle peak in the INTEGRAL pulse profile above $20 \mathrm{keV}$ is located at the same phase as one of the pulses detected below $10 \mathrm{keV}$. The second pulse detected with INTEGRAL is slightly shifted with respect to the second peak observed in the soft X-ray band. We performed consistent phase-resolved spectroscopy over the total high-energy band and identify at least three genuinely different pulse components with different spectra. The high level of consistency between the detailed results from the four missions is indicative of a remarkably stable geometry underlying the emission scenario. Finally, we discuss the derived detailed characteristics of the high-energy emission of 4U 0142+61 in relation to three models for the non-thermal hard X-ray emission.
\end{abstract}

Key words. stars: neutron - stars: pulsars: individual: 4U 0142+61 - gamma rays: observations

\section{Introduction}

Anomalous X-ray pulsars (AXPs) are a special subset of young isolated neutron stars with pulse periods in the range $\sim 2-12 \mathrm{~s}$ and with high period derivatives, on the order of $10^{-10}-10^{-12} \mathrm{~s} \mathrm{~s}^{-1}$. Their (anomalous) X-ray luminosities $\left(L_{\mathrm{X}} \sim\right.$ $10^{33}-10^{35} \mathrm{erg} \mathrm{s}^{-1}$ within $2-10 \mathrm{keV}$ ) are orders of magnitude too high to be explained by rotational energy released due to spin down. Different origins of energy budgets proposed to explain the high luminosities were not consistent with the observational characteristics of AXPs (cf. Woods \& Thompson 2006; Kaspi 2007, for a review and latest developments). However, for Soft Gamma-ray Repeaters (SGRs), which are another subset of young isolated X-ray pulsars with similar periods, a toroidal magnetic field in the neutron-star core as an energy reservoir was proposed by Duncan \& Thompson (1992). Their magnetar model for SGRs can explain the anomalous X-ray luminosities of AXPs by the decay of an ultra-high magnetic field $\left(B \gtrsim 10^{15} \mathrm{G}\right.$ ). The magnetar model also explains numerous other observable characteristics from AXPs like the large period derivatives and bursting behaviour. Most features of the magnetar model can be found in Duncan \& Thompson (1992); Thompson \& Duncan (1993, 1995, 1996); Thompson et al. (2002). Observational evidence collected over the last five years clearly shows that AXPs and SGRs have similar characteristics and therefore are now believed to be subsets of magnetars (Woods \& Thompson 2006).

In this paper we present new results for AXP 4U 0142+61. This particular AXP has a spin period of $P=8.69 \mathrm{~s}$ and a period derivative of $\dot{P}=0.20 \times 10^{-11} \mathrm{~s} \mathrm{~s}^{-1}$. The inferred surface magnetic field strength is $B=3.2 \times 10^{19} \sqrt{P \dot{P}} \mathrm{G}=1.3 \times 10^{14} \mathrm{G}$. In the $2-10 \mathrm{keV}$ band this is the brightest persistent AXP with a 
luminosity $L_{\mathrm{X}} \sim 1 \times 10^{35} \mathrm{erg} \mathrm{s}^{-1}$ assuming a distance of $3.6 \mathrm{kpc}$ (Durant \& van Kerkwijk 2006a).

A remarkable discovery with the hard X-ray/soft gamma-ray telescope INTEGRAL (Winkler et al. 2003) is the existence of a hard spectral component in the spectra of AXPs at energies above $20 \mathrm{keV}$. Molkov et al. (2004) reported hard X-ray emission (>20 keV) from the position of AXP 1E 1841-045 in the supernova remnant G27.4+0.0 (Kes 73). Kuiper et al. (2004) showed unambiguously that the hard X-rays indeed originate from the AXP and not from the supernova remnant by detecting pulsations in the hard X-ray emission from this source using the Rossi $X$-ray Timing Explorer (RXTE) PCA and HEXTE data.

INTEGRAL detections have been reported for two other AXPs. Revnivtsev et al. (2004) and den Hartog et al. (2004) detected 1RXS J170849-400910 (hereafter 1RXS J1708-40) and $4 \mathrm{U} 0142+61$, respectively. Also for these and for a fourth AXP, 1E 2259+586, Kuiper et al. (2006) discovered pulsed hard X-ray emission using RXTE PCA and HEXTE data.

The INTEGRAL total-emission spectra available for 1E 1841-045, 1RXS J1708-40 and 4U 0142+61 are all powerlaw like and hard. The spectra of these three AXPs have photon indices $^{1}$ of $1.32 \pm 0.11,1.44 \pm 0.45$ and $1.05 \pm 0.11$, respectively (Kuiper et al. 2006). Furthermore, den Hartog et al. (2007) performed a multi-frequency campaign in which $4 \mathrm{U} 0142+61$ was observed from radio to hard X-rays almost simultaneously. A dedicated INTEGRAL observation of $1 \mathrm{Ms}$ was part of this campaign. This observation yielded a power-law like spectrum up to $229 \mathrm{keV}$ with a photon index of $0.79 \pm 0.10$ with a luminosity of $2.6 \times 10^{35} \mathrm{erg} \mathrm{s}^{-1}$, which is roughly 2000 times the maximum luminosity possible by energy release due to spin down.

Interestingly, 4U 0142+61 exhibited some bursting activity discovered during the RXTE monitoring of this source. A first burst was found on April 6, 2006 (Kaspi et al. 2006). Soon thereafter, on June 25, four more bursts were detected (Dib et al. 2006). During these last events, the source seemed to be in an active state. Gavriil et al. (2007) discovered a very large X-ray burst on February 7, 2007. This burst was extraordinary as it showed characteristics similar to a SGR giant flare, only less energetic. It started with a bright and short spike with a peak flux of $\sim 2.5 \times 10^{-8} \mathrm{erg} \mathrm{cm}^{-2} \mathrm{~s}^{-1}(2-60 \mathrm{keV})$ and in the first part of the decaying tail single pulsations of the AXP were visible. The extended tail lasted up to half an hour. The fluence $\left(T_{90}\right)$ of this burst was $\sim 14.5 \times 10^{-8} \mathrm{erg} \mathrm{cm}^{-2} \mathrm{~s}^{-1}(2-60 \mathrm{keV})$, which is $6-88$ times higher than the previous five bursts from $4 \mathrm{U} 0142+61$. Moreover, the spectrum shows significant spectral features (Gavriil et al. 2008).

Theoretical attempts to find physical explanations for the hard X-ray emission have, so far, not been able to explain all of the published properties and for some aspects the data cannot discriminate between the proposed models. More detailed observational results are required to further constrain the geometries of the production sites as well as the allowed production processes. In the discussion (see, Sect. 5) we summarise the different theoretical approaches, promising progress, but also their shortcomings.

In this work, detailed hard X-ray characteristics are presented using all available INTEGRAL data for 4U $0142+61$ to date. These include total spectra (pulsed + DC emission), both time-averaged (whole mission) as well as in 5 shorter time stretches to look for possible long-term time variability. We also analysed two Target of Opportunity observations performed

\footnotetext{
${ }^{1}$ Defining the power law as $F=F_{0} \times E^{-\Gamma}$ with $F_{0}$ the normalisation in units of $\mathrm{ph} \mathrm{cm}^{-2} \mathrm{~s}^{-1} \mathrm{keV}^{-1}$ and $\Gamma$ the photon index.
}

shortly after the abovementioned burst activities to look for variability of any kind. Furthermore, the time-averaged total-pulsed spectrum as well as time-averaged phase-resolved pulsed spectra are presented.

In order to study possible relations between the hard $(>10 \mathrm{keV})$ and soft $(<10 \mathrm{keV})$ X-ray bands we have (re)analysed archival XMM-Newton, RXTE-PCA and ASCAGIS data applying parameters and selections consistent with the INTEGRAL analyses.

\section{Observations and analysis}

\subsection{INTEGRAL}

The hard X-ray/soft gamma-ray (3-8 MeV) mission INTErnational Gamma-Ray Astrophysics Laboratory (INTEGRAL; Winkler et al. 2003, has been operational since October 2002. For this work observations by both main instruments IBIS and SPI are used. IBIS, Imager on Board the INTEGRAL Satellite (Ubertini et al. 2003), is a coded-mask instrument with a low-energy detector called the INTEGRAL Soft Gamma-Ray Imager (ISGRI; Lebrun et al. 2003) which is sensitive between $\sim 20 \mathrm{keV}$ and $\sim 300 \mathrm{keV}$ and has a wide field of view (FOV) of $29^{\circ} \times 29^{\circ}$ (full-width zero response). The angular resolution is about 12'. The Spectrometer for INTEGRAL (SPI; Vedrenne et al. 2003) is also a coded-mask instrument which is sensitive between $\sim 20 \mathrm{keV}$ and $\sim 8 \mathrm{MeV}$. The FOV is $35^{\circ} \times 35^{\circ}$ (full-width zero response). This instrument is optimised for spectroscopy and has a modest angular resolution of $\sim 2.5$. SPI is of particular use in this work for its greater sensitivity to IBIS above $\sim 200 \mathrm{keV}$.

In its default operation modes, INTEGRAL observes the sky in dither patterns (Jensen et al. 2003). Typical pointings (Science Windows, ScWs) have integration times of $\sim 1800-3600 \mathrm{~s}$. Table 1 lists the total exposure time for the selected $\mathrm{ScWs}$ for which $4 \mathrm{U} 0142+61$ was within an angle of 14.5 from the pointing direction of IBIS, as well as the effective on-source exposure, which is reduced due to off-axis viewing angles. Due to the visibility windows of this part of the Galaxy for INTEGRAL operations, the observations of the prime targets form groups of consequentive orbital revolutions (Revs).

There are three dedicated observations of $4 \mathrm{U} 0142+61$. The first one was performed mid 2005 and lasted for $1 \mathrm{Ms}$ and two were shorter Target of Opportunity (ToO) observations which were triggered by the bursting activity detected with RXTE on June 25, 2006 and February 7, 2007.

The data were screened for Solar flares and erratic countrates due to the passages through the Earth's radiation belts. After screening, $1671 \mathrm{ScWs}$, resulting in $4.7 \mathrm{Ms}$ total exposure time, were available for the analyses.

\subsubsection{INTEGRAL spectra from spatial analysis}

For spectral analysis the IBIS-ISGRI data of all $1671 \mathrm{ScWs}$ were reduced using the Off-line Scientific Analysis (OSA) software package version 5.1 (see Goldwurm et al. 2003). For each ScW, deconvolved sky images were created in 20 energy intervals using exponential binning between $20 \mathrm{keV}$ and $300 \mathrm{keV}$. For each energy band, time averaged count rates were determined by averaging the count rates, weighted by the variances, from all deconvolved maps at the position of $4 \mathrm{U} 0142+61$, for each set of consecutive observations and the total data set. To convert the count rates to flux values we used the procedure described by Kuiper et al. (2006). The measured AXP count rates were normalized 
Table 1. Summary of the INTEGRAL observations of $4 \mathrm{U} 0142+61$. The following information is given: the revolution intervals; the observations time spans both in MJD as in calendar dates; the number of Science Windows; exposure times $\left(t_{\exp }\right)$; and the effective on-source exposure $\left(t_{\text {eff }}\right)$.

\begin{tabular}{lccccc}
\hline \hline Rev. & & Time span & ScWs & $t_{\text {exp }}(\mathrm{ks})$ & $t_{\text {eff }}(\mathrm{ks})$ \\
\hline $142-468$ & $52985-53960$ & Dec. 12, 2003-Aug. 13, 2006 & 1617 & 4494.4 & 2365.3 \\
\hline $142-189$ & $52985-53125$ & Dec. 12, 2003-Apr. 30, 2004 & 404 & 963.2 & 342.0 \\
$202-269$ & $53165-53367$ & Jun. 9, 2004-Dec. 28, 2004 & 410 & 976.5 & 501.6 \\
$331-336$ & $53550-53568$ & Jun. 29, 2005-Jul. 17, 2005 & 265 & 867.6 & 827.9 \\
$384-396$ & $53710-53745$ & Dec. 6, 2005-Jan. 10, 2006 & 435 & 1334.3 & 420.6 \\
$452-468$ & $53912-53960$ & Jun. 26, 2006-Aug. 13, 2006 & 103 & 353.8 & 273.2 \\
\hline 454 ToO & $53918-53920$ & Jul. 2, 2006-Jul. 4, 2006 & 59 & 213.7 & 209.7 \\
528 ToO & $54139-54142$ & Feb. 8, 2007-Feb. 11, 2007 & 54 & 198.6 & 195.5 \\
\hline
\end{tabular}

Table 2. Phase-coherent ephemerides derived from RXTE-PCA monitoring data, valid for the analysed INTEGRAL observations.

\begin{tabular}{llllllllr}
\hline \hline AXP & $\begin{array}{l}\text { Start } \\
{[\mathrm{MJD}]}\end{array}$ & $\begin{array}{l}\text { End } \\
{[\mathrm{MJD}]}\end{array}$ & $\begin{array}{l}\text { INTEGRAL } \\
\text { range (Revs) }\end{array}$ & $\begin{array}{l}t_{\text {Epoch }} \\
{[\mathrm{MJD}, \mathrm{TDB}]}\end{array}$ & $\begin{array}{l}v \\
{[\mathrm{~Hz}]}\end{array}$ & $\begin{array}{c}\dot{v} \\
\times 10^{-13}\left[\mathrm{~Hz} \mathrm{~s}^{-1}\right]\end{array}$ & $\begin{array}{c}\ddot{v} \\
\times 10^{-22}\left[\mathrm{~Hz} \mathrm{~s}^{-2}\right]\end{array}$ \\
\hline 4U 0142+61 & 52549 & 53169 & $001-203$ & 52726 & 0.1150945693297 & -0.267038 & 0.220 & 0.9442 \\
4U 0142+61 & 53251 & 53619 & $230-353$ & 53420 & 0.1150929854501 & -0.263899 & 0.307 & 0.8906 \\
4U 0142+61 & 53562 & 53745 & $334-396$ & 53650 & 0.1150924611500 & -0.267430 & -0.319 & 0.2817 \\
\hline
\end{tabular}

to the Crab count rates, determined from Crab observations performed during INTEGRAL Rev. 102. The AXP fluxes in Crab units are converted into photon fluxes using a curved power-law shape;

$$
F_{\gamma}=1.5703(14) \times\left(E_{\gamma} / 0.06335\right)^{-2.097(2)-0.0082(16) \times \ln \left(E_{\gamma} / 0.06335\right)}
$$

(Eq. (2) in Kuiper et al. 2006), where $F_{\gamma}$ is expressed in $\mathrm{ph} /\left(\mathrm{cm}^{2} \mathrm{~s} \mathrm{MeV}\right)$ and $E_{\gamma}$ in $\mathrm{MeV}$. Finally the spectra were imported into XSPEC version 12.3 (Arnaud 1996), which is used for the spectral fitting. All errors quoted in this paper are $1 \sigma$ errors, unless stated otherwise.

The spectral analysis of SPI data is based on all (screened) data up to Rev. 336. The data were binned in 9 energy bins covering the 20-1 MeV energy range. For each of the energy bins, source fluxes have then been extracted using a maximum likelihood fitting procedure that considers the instrumental response of the telescope (Knödlseder 2004). The measured flux is then normalized to the Crab spectrum that was obtained by fitting the SPI data of revolutions 43-45. Multiplication by the above-mentioned curved power-law Crab model gives the photon fluxes.

\subsubsection{INTEGRAL timing analysis}

For the INTEGRAL timing analysis, we followed the procedure described by Kuiper et al. (2006). We only considered ISGRI data for this purpose. Data up to Rev. 396 are used (see Table 1). Events are selected from non-noisy detector pixels that were/could be illuminated by the source through an open mask element with a greater illumination factor than $25 \%$. Instrumental, on-board processing and ground-station time delays are corrected for (Walter et al. 2003). The event arrival times at the space craft are converted to arrival times at the Solarsystem barycenter using the JPL DE200 Solar-system ephemeris and the source position measured with Chandra (Juett et al. 2002). The barycentered arrival times are folded on an appropriate phase-connected ephemeris (see Table 2) created using contemporaneous RXTE data (see Sect. 2.2). The (TDB) time to pulse phase conversion taking into account consistent phase alignment for each ephemeris is provided by the following formula:

$$
\Phi(t)=v \cdot\left(t-t_{\text {Epoch }}\right)+\frac{1}{2} \dot{v} \cdot\left(t-t_{\text {Epoch }}\right)^{2}+\frac{1}{6} \ddot{v} \cdot\left(t-t_{\text {Epoch }}\right)^{3}-\Phi_{0} .
$$

The detection significances of the derived pulse profiles are estimated applying the $Z_{n}^{2}$ test (Buccheri et al. 1983).

In order to derive the spectrum of the pulsed emission, first the number of excess counts in the obtained pulse profiles above flat background/DC levels have to be determined. For this purpose, the pulse profiles for the selected energy bands are fitted with truncated Fourier series using two harmonics. The minimum of this fit is defined as the DC level. The excess counts are counted above this level and converted into flux units applying the same procedure for the Crab pulsar and normalising to the known Crab-pulsar spectrum (Eq. (3) in Kuiper et al. 2006)

$$
F_{\gamma}=0.4693(21) \times\left(E_{\gamma} / 0.04844\right)^{-1.955(7)-0.0710(78) \times \ln \left(E_{\gamma} / 0.04844\right)} \text {. }
$$

Phase-resolved spectra are similarly created using only excess counts within selected phase intervals (see Sect. 3.3).

\section{2. $R X T E$}

The Rossi X-ray Timing Explorer (RXTE) has been operational since 1996. For this work we use data from the Proportional Counter Arrays (PCA) onboard RXTE (Jahoda et al. 1996), which is a non-imaging instrument sensitive in the $2-60 \mathrm{keV}$ energy range. The FOV is approximately $1^{\circ}(F W H M)$.

Regular monitoring observations of $4 \mathrm{U} 0142+61$ with the RXTE-PCA (Gavriil \& Kaspi 2002) are the basis of the timing results in this paper. These observations allow us to create accurate phase-connected timing solutions. We have generated three new phase-connected ephemerides with consistent pulse alignment to cover the whole INTEGRAL-time span. The details of the ephemerides can be found in Table 2. They are in agreement with the solution presented by Dib et al. (2007), who also present a phase-coherent timing solution over the period 1996-2006.

We have used the same RXTE-PCA data as listed in Table 1 of Kuiper et al. (2006) which were taken between March 1996 and September 2003 before the INTEGRAL observations, to perform spectral timing analyses analogous to the procedures outlined by Kuiper et al. (2006). We note that in the folding 
Table 3. XMM-Newton observations of $4 \mathrm{U} 0142+61$. The observations are designated $\mathrm{A}, \mathrm{B}$ and $\mathrm{C}$ and referenced accordingly in the text. The observation dates, exposures and Good Time Interval (GTI) exposures for MOS and PN are given.

\begin{tabular}{llllll}
\hline \hline$\#$ & Obs ID & Date & $\begin{array}{l}\text { Exp. } \\
(\mathrm{ks})\end{array}$ & $\begin{array}{l}\text { GTI-MOS } \\
(\mathrm{ks})\end{array}$ & $\begin{array}{l}\text { GTI-PN } \\
(\mathrm{ks})\end{array}$ \\
\hline $\mathrm{A}$ & 0112781101 & $2003-01-24$ & 6.4 & 5.4 & 3.7 \\
$\mathrm{~B}$ & 0206670101 & $2004-03-01$ & 46.5 & 36.4 & 27.3 \\
$\mathrm{C}$ & 0206670201 & $2004-07-25$ & 23.9 & 23.0 & 20.6 \\
\hline
\end{tabular}

process of PCA data the application of ephemeris entry one of Table 2 results in pulse profiles that are shifted 0.543 in phase with respect to the profiles shown in Kuiper et al. (2006) for $4 \mathrm{U} 0142+61$.

Irrespective of the energy band, the pulse-profiles can be described sufficiently accurately by truncated Fourier series with 3 harmonics above constant background levels. The excess (pulsed) counts above the background levels are converted into flux units using properly PCU exposure weighted energy response matrices (see e.g. Sect. 3.2 of Kuiper et al. 2006).

We have extracted a time-averaged total-pulsed spectrum and phase-resolved spectra adopting the same phase intervals as selected for INTEGRAL (see Sect. 3.3). To derive unabsorbed (soft) X-ray spectra a Galactic Hydrogen absorption column of $N_{\mathrm{H}}=0.57 \times 10^{22} \mathrm{~cm}^{-2}$ has been used (as fixed value) as determined from spectral analyses of XMM-Newton observations (see Sect. 3.1.4).

\subsection{XMM-Newton}

XMM-Newton (Jansen et al. 2001) has been operational since 2000. Onboard are three CCD cameras for X-ray imaging, namely two EPIC (European Photon Imaging Camera) MOS cameras (Turner et al. 2001) and one EPIC-PN camera (Strüder et al. 2001). All cameras have a FOV of $\sim 30^{\prime}$ and are sensitive in the energy range $\sim 0.3-12 \mathrm{keV}$.

We have (re)analysed the four publicly available data sets acquired by XMM-Newton between 2002 and 2004. The data set taken on February 13, 2002 (Obs Id 0112780301) did not contain usable data for this work. The summary of the remaining 3 data sets can be found in Table 3 . We designate these observations $\mathrm{A}, \mathrm{B}$ and $\mathrm{C}$, respectively. In this work we present only the results using the PN. We have also analysed the data from both MOS instruments and used this for consistency checks. The PN was in small-window mode during observation A and in timing mode during observations B and C. In parallel studies Rea et al. (2007a) and Gonzalez et al. (2008) also analysed these XMM-Newton observations. Gonzalez et al. (2008) also included private XMM-Newton data taken in 2006 and 2007 together with Chandra and Swift data to study long-term variations below $10 \mathrm{keV}$.

The three data sets in Table 3 were analysed using SAS v. 7.0 and the latest calibration files that were available (Jan. 2007). The data were checked for solar (soft proton) flares by creating a light curve for each observation for events with energies larger than $10 \mathrm{keV}$. From these light curves the count-rate distribution has been determined and subsequently fitted with a Gaussian. Good Time Intervals (GTIs) were created by allowing only time stamps from time intervals for which the count rate was lower than the fitted mean count rate plus three times the width of the distribution $(3 \sigma)$. For observations $\mathrm{A}, \mathrm{B}$ and $\mathrm{C}$ these cutoff values were $0.082,0.243$ and 0.358 counts per second. PN single and double events, i.e. patterns less than and equal four, and an energy range of 0.3 to $12.0 \mathrm{keV}$ were selected. The arrival times of the selected events are barycentered adopting the most accurate celestial position of $4 \mathrm{U} 0142+61$ (Juett et al. 2002).

There was no need to correct for pile up in observation $\mathrm{A}$ as the count rate from $4 \mathrm{U} 0142+61$ was sufficiently low for the small-window mode. A circular extraction region centered at $4 \mathrm{U} 0142+61$ with a radius of $40^{\prime \prime}$ was used. For the background extraction two circular source-free regions centered at different locations in the small-window were chosen with radii of $35^{\prime \prime}$ and 47. , 5 , respectively.

Observations B and $\mathrm{C}$ were taken in timing mode. For these observations we chose extraction regions with a total width of 27 pixels centered on the source, which corresponds to $110^{\prime} .7$ close to the $95 \%$ encircled energy radius of the source. While the sensitive area (ARF) generator corrects for the remaining $\sim 5 \%$ of missed energy outside the source extraction region, it does not compensate for the subtraction of any source contribution in the chosen background extraction strip. We took a strip 11 pixels wide starting 102'. 5 from the point-spread function (PSF) core. The source contribution from this part of the PSF can be neglected. We fitted a Lorentzian plus constant background to the observed one-dimensional source profile. The source contribution in the 11 pixel wide background strip turned out to be a mere $0.48 \%$ of the normalized PSF, which means, taking into account the source-to-background scaling of 27/11, an ignored correction of about $1.2 \%$.

The extracted spectra have been binned, oversampling the energy resolution by a factor of three and then occasionally rebinned to ensure a minimum of 25 counts per bin.

For the XMM-Newton timing analysis, the events selected as described above are used. These events are folded using the appropriate ephemeris in Table 2. For the pulsed spectra the pulsed excess counts are extracted using the same procedure as described in Sect. 2.1.2 for the INTEGRAL pulsed spectrum, fitting the pulse profiles with truncated Fourier series with the first three harmonics above a constant background.

\subsection{ASCA}

ASCA (Tanaka et al. 1994) was operational for eight years from 1993. Onboard ASCA was the Gas Imaging Spectrometer (GIS, Ohashi et al. 1996; Makishima et al. 1996). The GIS was sensitive between $\sim 0.7-12 \mathrm{keV}$ and had a FOV of $\sim 50^{\prime}$.

We have revisited the spectral timing analysis by Kuiper et al. (2006) of the ASCA-GIS observation of 4U 0142+61 taken in July/August 1999 to make it consistent with this work, i.e., in the current spectral analysis we have changed and fixed the Galactic absorption column to the value derived from the XMM-Newton data (see Sect. 3.1.4). In the timing analysis the pulse phase has been redefined to be consistent with that of INTEGRAL, RXTE and XMM-Newton, allowing the same phase selections for the phase-resolved spectroscopy.

\section{Results}

In this section, we first present the INTEGRAL and XMM-Newton total spectra and the search for long-term variability followed by pulse profiles from our timing analyses of INTEGRAL, XMM-Newton, RXTE and ASCA. Finally, for the same instruments the total pulsed and phase-resolved spectra are derived consistently, revealing that genuinely different components contribute to the total pulsed emission. 


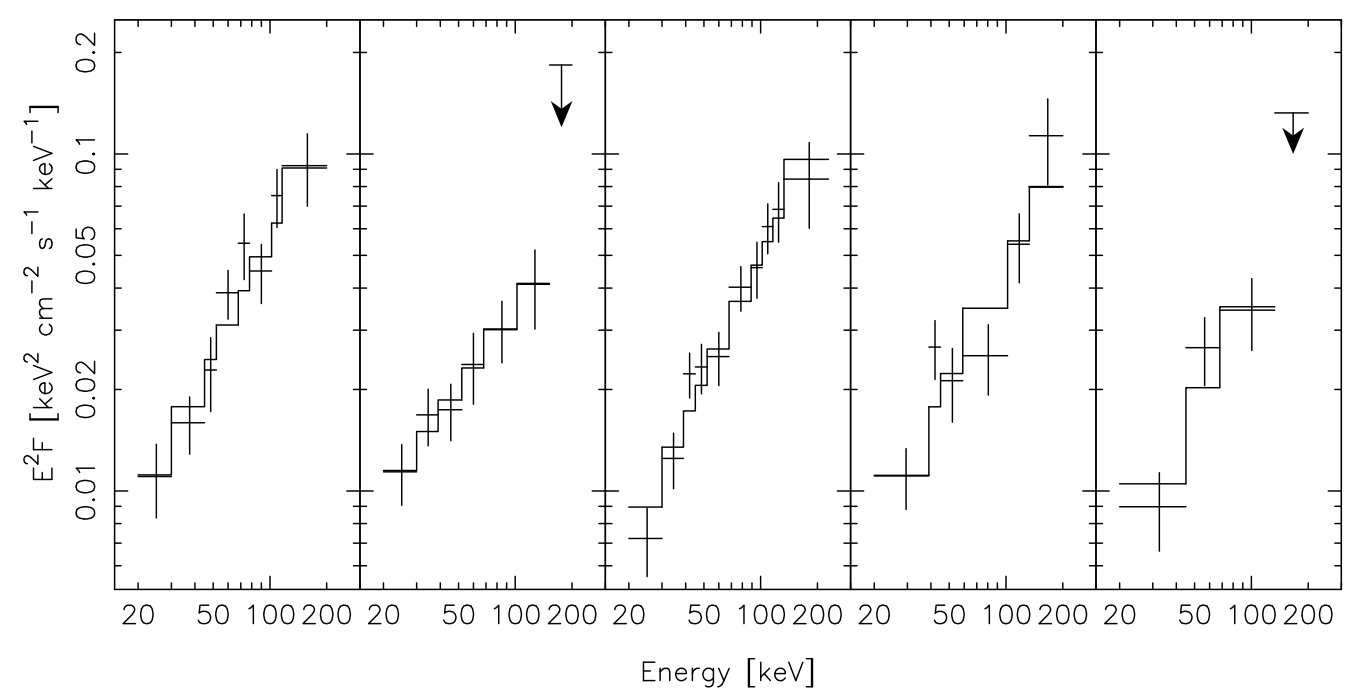

Fig. 1. Total-flux spectra of 4U0142+61 taken during INTEGRAL revolutions (from left to right); 142-189, 202-269, 331-336, 384-396 and 452-468. Data points are binned to $4 \sigma$ if the single bins are of less significance. The upper limits represent $2 \sigma$ confidence levels.

\subsection{Total spectrum and long-term variability}

Previous INTEGRAL studies by den Hartog et al. (2006, 2007) and Kuiper et al. (2006) show that hard X-rays from 4U 0142+61 have been detected at different epochs at similar flux levels, suggesting a stable and persistent hard X-ray spectrum. First, we will verify the stable nature of the high-energy emission. Then, we will present the time-averaged total spectrum using contemporaneous IBIS-ISGRI and SPI data. Finally, the total spectra of XMM-Newton are derived and an example is shown together with the INTEGRAL time-averaged total spectrum, rendering a broad-band X-ray view of the total spectrum.

\subsubsection{INTEGRAL ISGRI persistent spectra and long-term variability}

The ISGRI data were grouped in five sets as indicated in Table 1 and analysed as described in Sect. 2.1.1. The statistical quality is not equal for every spectrum, but $4 \mathrm{U} 0142+61$ is detected significantly in each group and spectra can be extracted from each data set. The resulting five total spectra are displayed in Fig. 1.

To fit the spectra we use single power-law models. As in previous work they describe the spectral shapes at a satisfactory level. The fit results for these spectra are listed in the upper part of Table 4 . The photon indices $\Gamma$ range from $0.79 \pm 0.10$ to $1.21 \pm 0.16$ and the fluxes in the $20-150 \mathrm{keV}$ energy band $\left(F_{20-150}\right)$ range from $7.8 \times 10^{-11}$ to $11.3 \times 10^{-11} \mathrm{erg} \mathrm{cm}^{-2} \mathrm{~s}^{-1}$.

The errors in the indices $\Gamma$ and fluxes $F_{20-150}$ are not independent. In order to check for possible long-term time variability we therefore compared the error contours of the different fits with respect to the error contours of the fit to the time-averaged total spectrum (see below in Sect. 3.1.2). The spectra extracted for Revs. 142-189 and 202-269 show the most deviation from the time-averaged spectrum. In Fig. 2 the error contours for these spectra are shown together with the best-fit values for the timeaveraged total spectrum (Revs. 142-468). The two spectra deviate from the average around the $\sim 2 \sigma$ level.

The standard deviations $\left(s=\sqrt{1 /(n-1) \sum_{i}\left(x_{i}-\bar{x}\right)^{2}}\right)$ for the power-law indices and the $20-150 \mathrm{keV}$ fluxes relative to

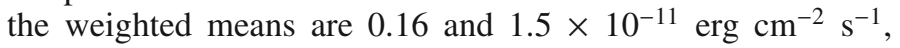

Table 4. Summary of the single power-law fits for the INTEGRALISGRI total-spectra of $4 \mathrm{U} 0142+61$ at different epochs for the observations summarized in Table 1 . For each epoch the photon index, $20-150 \mathrm{keV}$ flux and $\chi_{r}^{2}$ are given. For the the spectrum taken in Rev. $142-468$ the $20-229 \mathrm{keV}$ flux is also given.

\begin{tabular}{lccc}
\hline \hline Rev. & $\Gamma$ & $\begin{array}{c}F_{20-150 \times 10^{-11}} \\
{\left[\mathrm{erg} \mathrm{cm}^{-2} \mathrm{~s}^{-1}\right]}\end{array}$ & $\chi_{r}^{2}$ (d.o.f.) \\
\hline $142-189$ & $0.85 \pm 0.14$ & $11.34 \pm 0.78$ & $0.69(15)$ \\
$202-269$ & $1.21 \pm 0.16$ & $7.80 \pm 0.83$ & $0.66(13)$ \\
$331-336$ & $0.79 \pm 0.10$ & $9.80 \pm 0.57$ & $1.12(16)$ \\
$384-396$ & $0.89 \pm 0.17$ & $9.46 \pm 0.91$ & $0.91(15)$ \\
$452-468$ & $0.96 \pm 0.25$ & $7.8 \pm 1.2$ & $0.82(12)$ \\
\hline $454 \mathrm{ToO}$ & $1.06 \pm 0.30$ & $7.8 \pm 1.4$ & $0.98(12)$ \\
$528 \mathrm{ToO}$ & $0.88 \pm 0.34$ & $9.3 \pm 1.8$ & $1.63(11)$ \\
\hline $142-468$ & $0.93 \pm 0.06$ & $9.09 \pm 0.35^{\dagger}$ & $0.94(16)$ \\
$F_{20-229}=(15.01 \pm 0.82) \times 10^{-11} \mathrm{erg} \mathrm{cm}^{-2} \mathrm{~s}^{-1}$ \\
\hline \multicolumn{4}{c}{} \\
\hline
\end{tabular}

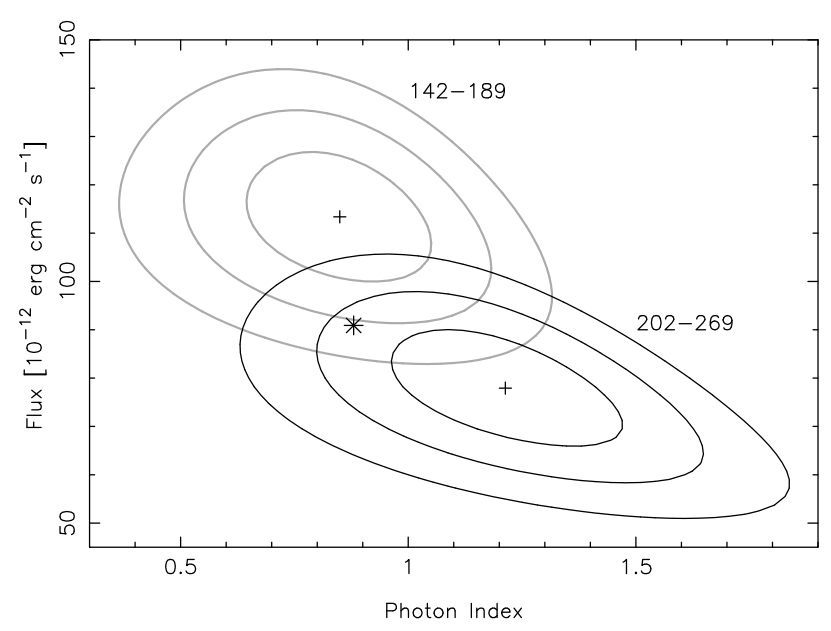

Fig. 2. Error contours $(1 \sigma, 2 \sigma$ and $3 \sigma)$ of observations 142-189 (in grey) and 202-269 (in black) with respect to the time-averaged measurement (star).

respectively. Therefore, both the power-law shape and the $20-150 \mathrm{keV}$ flux of $4 \mathrm{U} 0142+61$ are stable within $17 \%(1 \sigma)$. 


\subsubsection{INTEGRAL ISGRI and SPI total spectra}

Using all available (Revs. 142-468, see Table 1) 2.37 Ms net on-source exposures the time-averaged total spectrum (Fig. 3) derived from ISGRI data can be fitted satisfactorily with a single power-law model. The best fit parameters give a photon index $\Gamma=0.93 \pm 0.06$ and a $20-150 \mathrm{keV}$ flux of $(9.09 \pm 0.35) \times$ $10^{-11} \mathrm{erg} \mathrm{cm}^{-2} \mathrm{~s}^{-1}$. 4U $0142+61$ has been detected up to $229 \mathrm{keV}$ with a $4.3 \sigma$ significance in the $152-229 \mathrm{keV}$ band.

Even though the upper limits at MeV energies obtained with the Compton telescope COMPTEL onboard CGRO (den Hartog et al. 2006, also shown in Fig. 3) strongly suggest that the spectrum has to break at a few hundred $\mathrm{keV}$, the ISGRI spectrum shows no indication of a spectral break. The quality of the fit $\left(\chi_{r}^{2}=0.94\right.$; d.o.f. $\left.=16\right)$ assuming a single power-law model over the 20-300 keV range is good and the use of a more complex model with additional parameters is statistically not justified.

SPI is more sensitive than ISGRI at energies higher than $\sim 200 \mathrm{keV}$. Therefore, we have also analysed all available SPI data with $4 \mathrm{U} 0142+61$ in the FOV. We have extracted the SPI spectrum and detected 4U 0142+61 significantly above $40 \mathrm{keV}$ up to $140 \mathrm{keV}$. The maximum detection significance is $5 \sigma$ between $80 \mathrm{keV}$ and $140 \mathrm{keV}$. In the next energy bin $(140-220 \mathrm{keV})$ a marginal detection of $2.6 \sigma$ is achieved. The six flux values between 20 and $220 \mathrm{keV}$ can be fitted $\left(\chi_{r}^{2}=1.67\right.$; d.o.f. $=4)$ with a power-law model with photon index $\Gamma=0.86 \pm$ 0.20 and a $20-150 \mathrm{keV}$ flux of $(8.8 \pm 1.2) \times 10^{-11} \mathrm{erg} \mathrm{cm}^{-2} \mathrm{~s}^{-1}$, which is consistent with the ISGRI total spectrum (see Fig. 3).

Fitting the SPI spectrum with a single power law using all 9 spectral bins between 20 and $1000 \mathrm{keV}$ we find a poor and unsatisfactory fit with a $\chi_{r}^{2}=2.57$ for 7 d.o.f. $\left(\chi^{2}=18\right)$. Therefore, we followed Kuiper et al. (2006), by using a logparabolic function;

$F=F_{0} \times\left(\frac{E}{E_{0}}\right)^{-\alpha-\beta \cdot \ln \left(\frac{E}{E_{0}}\right)}$

with $E_{0}$ (in units $\mathrm{keV}$ ) the pivot energy introduced to minimize correlations between the parameters, and $F_{0}$ the flux (in units ph $\mathrm{cm}^{-2} \mathrm{~s}^{-1} \mathrm{keV}^{-1}$ ) at $E_{0}$. Note that this function is a simple power law if the curvature parameter $\beta$ is equal to zero.

The fit of the SPI spectrum improves with $\Delta \chi_{1}^{2}=15.77$, which corresponds to a $\sim 4 \sigma$ improvement for one additional fit parameter.

To exploit all available spectral information we fitted the ISGRI and SPI spectra simultaneously. Starting from a single power-law model we obtained a fit with a $\chi_{r}^{2}=1.58$ for 27 d.o.f. $\left(\chi^{2}=42.8\right)$ which can be improved significantly. The model described by this power-law is too high in the energy range with the SPI upper limits i.e. above $220 \mathrm{keV}$. Again using a logparabolic function an optimum fit with a $\chi^{2}$ of 20.1 is achieved. A $\Delta \chi_{1}^{2}$ of 22.7 for one additional parameter gives a $4.8 \sigma$ fit improvement. This is the first clear detection of the spectral break in the total spectrum of $4 \mathrm{U} 0142+61$ above $20 \mathrm{keV}$ using contemporaneous high-energy data. The best fit parameters are $\alpha=1.26 \pm 0.09, \beta=0.41 \pm 0.09$ and $F_{0}=(5.1 \pm 0.3) \times$ $10^{-6} \mathrm{ph} \mathrm{cm}^{-2} \mathrm{~s}^{-1} \mathrm{keV}^{-1}$ at $E_{0}=91.527 \mathrm{keV}$. The peak energy (for a $v F_{v}$ representation, equivalent to $E^{2} F$ ) corresponding to these parameters is $E_{\text {peak }}=228_{-53}^{+106} \mathrm{keV}$, while the $20-150 \mathrm{keV}$ flux is $(8.97 \pm 0.86) \times 10^{-11} \mathrm{erg} \mathrm{cm}^{-2} \mathrm{~s}^{-1}$ (fitting three free parameters).

Including the non-contemporaneous COMPTEL (0.75$30 \mathrm{MeV}$ ), flux measurements (upper limits in Fig. 3) in a similar

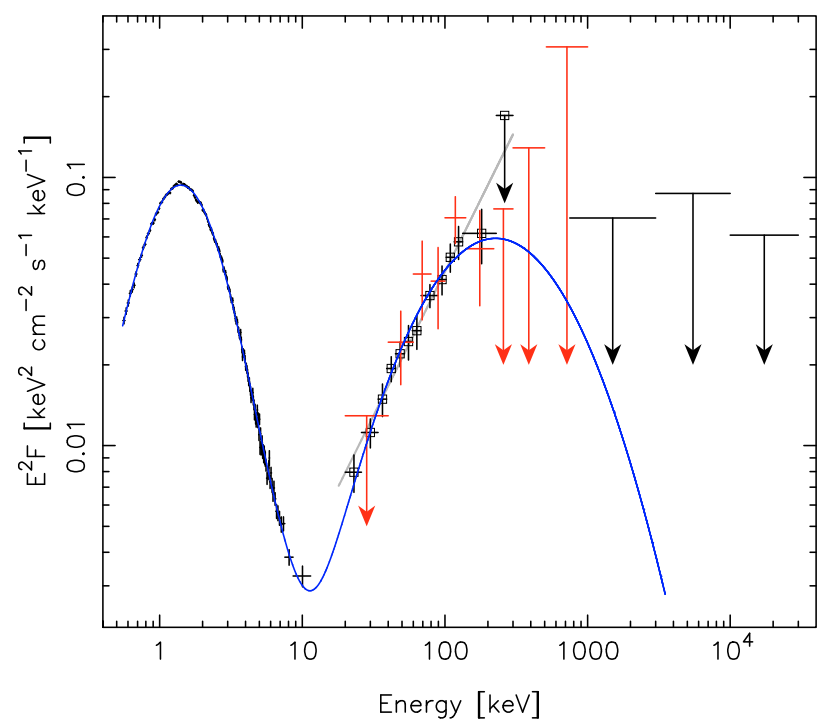

Fig. 3. Unabsorbed total spectra of $4 \mathrm{U} 0142+61$ as measured with different instruments; XMM-Newton (Obs B; $0.55-11.5 \mathrm{keV})$ in black; INTEGRAL-ISGRI (Revs. 142-468; 20-300 keV) in black with open square symbols; INTEGRAL SPI (20-1000 keV) in red; and CGRO COMPTEL (0.75-30 MeV) limits in black. Shown are also the best single power-law model fit for the ISGRI data-points (in grey) and the "three logparabola" fit for the whole band (in blue). See Sect. 3.1 for details.

fit yielded best fit parameters with somewhat smaller uncertainties. We found as best fit parameters: $\alpha=1.484 \pm 0.057, \beta=$ $0.351 \pm 0.044$ and $F_{0}=(3.01 \pm 0.16) \times 10^{-6} \mathrm{ph} \mathrm{cm}^{-2} \mathrm{~s}^{-1} \mathrm{keV}^{-1}$ at $E_{0}=133.718 \mathrm{keV}$. The peak energy $\left(E^{2} F\right)$ corresponding to these parameters is $E_{\text {peak }}=279_{-41}^{+65} \mathrm{keV}$. We note that this result is based on an assumed "logparabolic" spectral shape. We adopted this spectral form in order to comply with the SPI and COMPTEL limits. However, we do not claim that we have measured the spectral shape above $\sim 200 \mathrm{keV}$ in detail. In particular, the extension of the spectrum towards $\mathrm{MeV}$ energies is uncertain. Significant measurements in the energy range between $200 \mathrm{keV}$ and $1 \mathrm{MeV}$ are required to unravel the true spectral shape in the vicinity of the break energy.

\subsubsection{INTEGRAL post-burst spectra}

Of the five AXPs for which bursts have been detected, $4 \mathrm{U} 0142+61$ is so far the only one for which a hard X-ray spectrum above $\sim 10 \mathrm{keV}$ has been observed. After two remarkably different bursting events detected with RXTE-PCA we requested two INTEGRAL ToO observations in order to study possible correlations between the soft- and hard X-ray emission. For both occasions $\sim 200 \mathrm{ks}$ ToO time was granted. The source was observed in Revs. 454 and 528 (see Table 1).

We have detected $4 \mathrm{U} \quad 0142+61$ with detection significances in the $20-150 \mathrm{keV}$ energy band of $5.4 \sigma$ and $5.9 \sigma$ for Rev-454 and Rev-528, respectively. Both spectra have been fitted with a power-law model which described the spectra well. The fit parameters for the first $\mathrm{ToO}$ are $\Gamma=1.06 \pm 0.30$ and $F_{20-150}=(7.8 \pm 1.4) \times 10^{-11} \mathrm{erg} \mathrm{cm}^{-2} \mathrm{~s}^{-1}$ and for the 2 nd ToO the fit parameters are $\Gamma=0.88 \pm 0.34$ and $F_{20-150}=(9.3 \pm 1.8) \times 10^{-11} \mathrm{erg} \mathrm{cm}^{-2} \mathrm{~s}^{-1}$. The values are fully 
Table 5. XMM-Newton EPIC-PN spectral fits of the total-emission spectrum of $4 \mathrm{U} 0142+61$. Three logparabolae (Eq. (4)) were used for an empirical fit including three fixed parameters from the ISGRI/SPI 20-1000 keV spectral fit. For each observation (A, B and C, see Table 3) six optimum parameter values are shown, three for each logparabolic model (indicated with a subscript number), for the best fit $(0.55-10 \mathrm{keV})$. The last row lists the unabsorbed $2-10 \mathrm{keV}$ energy flux.

\begin{tabular}{lccc}
\hline \hline & $\mathrm{A}$ & $\mathrm{B}$ & $\mathrm{C}$ \\
\hline$F_{0,1}$ & $(6.85 \pm 0.05) \times 10^{-2}$ & $(5.50 \pm 0.02) \times 10^{-2}$ & $(4.57 \pm 0.02) \times 10^{-2}$ \\
$\alpha_{1}$ & $0.933 \pm 0.012$ & $0.673 \pm 0.006$ & $0.528 \pm 0.008$ \\
$\beta_{1}$ & $1.534 \pm 0.013$ & $1.899 \pm 0.007$ & $2.089 \pm 0.010$ \\
$F_{0,2}$ & $(1.04 \pm 0.04) \times 10^{-2}$ & $(2.33 \pm 0.02) \times 10^{-2}$ & $(3.20 \pm 0.02) \times 10^{-2}$ \\
$\alpha_{2}$ & $2.095 \pm 0.041$ & $1.671 \pm 0.009$ & $1.440 \pm 0.008$ \\
$\beta_{2}$ & $0.212 \pm 0.025$ & $0.622 \pm 0.007$ & $0.808 \pm 0.006$ \\
$F_{2-10 \mathrm{keV}}\left(\mathrm{erg} \mathrm{cm}^{-2} \mathrm{~s}^{-1}\right)$ & $(6.68 \pm 0.40) \times 10^{-11}$ & $(6.27 \pm 0.14) \times 10^{-11}$ & $(6.49 \pm 0.15) \times 10^{-11}$ \\
\hline
\end{tabular}

a) The photon fluxes $F_{0,1}$ and $F_{0,2}$ are expressed in $\mathrm{ph} \mathrm{cm}^{-2} \mathrm{~s}^{-1} \mathrm{keV}^{-1}$ at $E_{0}=1 \mathrm{keV}$.

b) Error estimates $(1 \sigma)$ for the fit parameters are obtained by fixing all parameters except the parameter of interest at their optimum values and subsequently determining where the parameter of interest reaches a $\Delta \chi_{6-1}^{2}$ step of 7.038 from the global minimum. This increment corresponds to a $1 \sigma$ step for $6-1$ degrees of freedom.

consistent with those for the time-averaged spectrum ${ }^{2}$ (see also Table 4).

Gonzalez et al. (2008) reported a flux increase of $(15 \pm 3) \%$ in the $2-10 \mathrm{keV}$ band observed with XMM-Newton coinciding with the burst activity in 2006-2007. It should be noted, however, that in order to measure with INTEGRAL a significant $(>3 \sigma)$ change in the hard X-ray spectrum within a $200 \mathrm{ks}$ observation, the flux level should have changed by at least $\sim 60 \%$. Correlated changes in the hard and soft X-ray fluxes of the scale reported by Gonzalez et al. (2008) could therefore not be measured.

\subsubsection{XMM-Newton EPIC-PN total emission spectra}

We derived the XMM-Newton EPIC-PN total (=pulsed plus DC) spectra as described in Sect. 2.3 (see e.g. Fig. 3 for an example). We first investigated the absorption column $\left(N_{\mathrm{H}}\right)$ using the XMM observations with the best statistics ( $\mathrm{B}$ and $\mathrm{C}$ ) and concentrated on energies below $\sim 5 \mathrm{keV}$ i.e. the soft part of the $\mathrm{X}$-ray spectrum.

We used logparabolic functions in order for the spectra to bend downwards to the optical regime, as required by the broadband spectrum (see e.g. the spectrum from the first broad-band campaign by den Hartog et al. 2007). If a traditional model, a combination of a black body and a power law, was chosen, the soft power-law component would keep increasing towards lower energies, which can only be compensated for by increasing the absorption column. The latter would be forced to unrealistically high values (see e.g. Rea et al. 2007a, who derived $\left.N_{\mathrm{H}}=(1.00 \pm 0.01) \times 10^{22} \mathrm{~cm}^{-2}\right)$. Fixing the $N_{\mathrm{H}}$ to the value published by Durant \& van Kerkwijk $\left(2006 \mathrm{~b}, N_{\mathrm{H}}=(0.64 \pm 0.07) \times\right.$ $10^{22} \mathrm{~cm}^{-2}$ ) from an accurate analysis of data of the Reflection Grating Spectrometer aboard XMM-Newton yielded poor fits at low energies between $\sim 0.6 \mathrm{keV}$ and $\sim 1 \mathrm{keV}$ in both observations $\mathrm{B}$ and $\mathrm{C}$. Therefore, we treated $N_{\mathrm{H}}$ as a free parameter. For both observations we find an absorption column of $(0.57 \pm 0.02) \times 10^{22} \mathrm{~cm}^{-2}$, which is somewhat lower than, but consistent with the value obtained by Durant \& van Kerkwijk (2006b). Therefore, assuming that the origin of the absorption is Galactic and thus constant, we fix $N_{\mathrm{H}}$ to $0.57 \times 10^{22} \mathrm{~cm}^{-2}$ for the

\footnotetext{
2 Note that the 1 st ToO is included in the time-averaged total spectrum, but we have tested the ToO with the time-averaged total spectrum of the first four data sets from which it was not statistically different.
}

rest of the XMM-Newton analyses. Güver et al. (2008) find similar values for $N_{\mathrm{H}}$ by applying a surface thermal-emission and magnetosphere scattering model to the same XMM-Newton data in a parallel analysis.

Next, we consider the total XMM-Newton spectral energy window up to $\sim 12 \mathrm{keV}$. An additional model component is required to describe at least a part $(<20 \%)$ of the harder $(\sim 5-12 \mathrm{keV}) \mathrm{X}$-ray emission from both observations B and C. For this component a second logparabolic function is introduced, instead of the traditionally introduced power-law component. Furthermore, a third logparabolic component with fixed model parameters prescribed by the ISGRI/SPI joint fit above $20 \mathrm{keV}$ (see Sect. 3.1.2) contributes in this energy band and is included in the total fit. Without the need to introduce an XMM-NewtonISGRI inter-calibration factor, a good fit was obtained to the high-energy parts of the XMM-Newton spectra. In Table 5 the fit results are presented for the three XMM-Newton observations.

However, for both long observations (obs. B and C), statistically unacceptable fits are obtained employing this empirical approach. Significant residuals are apparent below $2 \mathrm{keV}$, because the logparabolae cannot follow the spectral shape exactly at these low energies. The small deviations from the optimum fit (hardly visible in Fig. 3) are of the order of a few percent for energies below $2 \mathrm{keV}$. We use the spectral fits, however, primarily for the conversion of absorbed fluxes into unabsorbed fluxes (data points shown in Fig. 3) by calculating for each energy bin the fraction of energy that is absorbed. In Sect. 3.3 we use these high-statistics, unabsorbed total flux measurements in even broader bins to derive the pulsed fraction as a function of energy, and the uncertainty in that analysis is dictated by the errors in the fluxes from the phase resolved analysis. Therefore, the empirical two plus one (fixed) logparabolae fits assuming a constant absorption column of $0.57 \times 10^{22} \mathrm{~cm}^{-2}$ are sufficiently accurate for our purposes.

Note from Table 5 that the total unabsorbed $2-10 \mathrm{keV}$ energy flux is consistent with being constant during the time span of the three XMM-Newton observations. This is in agreement with Rea et al. (2007a) and Gonzalez et al. (2008), however, Gonzalez et al. (2008) report a flux increase of $(15 \pm 3) \%$ $(2-10 \mathrm{keV})$ between the epochs of our XMM-Newton observations $\mathrm{A}, \mathrm{B}$ and $\mathrm{C}$ and the epochs after the bursting activities started in 2006. The flux levels around $10 \mathrm{keV}$ of the three XMM-Newton spectra are rather similar. Figure 3, showing only the XMM-Newton obs. B spectrum, is therefore a good 


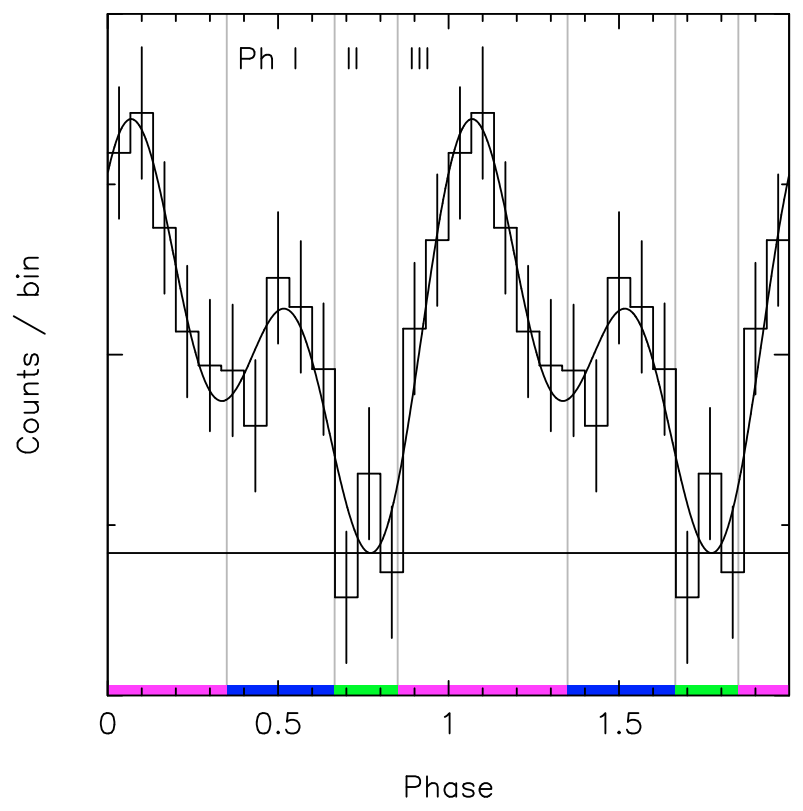

Fig. 4. 20-160 keV ISGRI pulse profile of $4 \mathrm{U} 0142+61$. The significance is $6.5 \sigma$ using a $Z_{2}^{2}$ test (fit shown as solid curve). The fitted DC level is indicated with a horizontal line. Also indicated are the phase intervals for Ph I, II and III (see Table 6) with vertical grey lines and with colours.

representation of the total high-energy ( $>0.5 \mathrm{keV})$ spectrum, particularly showing the abrupt transition from soft to hard $\mathrm{X}$-rays.

\subsection{Timing analysis: pulse profiles}

In this section we first present the time-averaged INTEGRAL IBIS-ISGRI pulse profiles of $4 \mathrm{U} 0142+61$. Then, we examine the XMM-Newton EPIC-PN and ASCA-GIS pulse profiles in single observations and test these for variability with time and energy, respectively. Finally, we compare the time-averaged ISGRI pulse profiles with the time-averaged XMM-Newton pulse profiles.

\subsubsection{INTEGRAL ISGRI pulse profiles}

Using the procedure described in Sect. 2.1.2 it is possible to detect significant pulsed emission from 4 U $0142+61$ in the ISGRI data. For the energy range $20-160 \mathrm{keV}$ a maximum significance of $6.5 \sigma$ is reached using a $Z_{2}^{2}$ test. The $20-160 \mathrm{keV}$ pulse profile, shown in Fig. 4, is broad and appears to be double peaked with a DC level in a narrow phase interval of width $\sim 0.2$.

Pulse profiles for the differential energy intervals $20-50 \mathrm{keV}$ and 50-160 keV are shown in Figs. 7E, F. The significances of the ISGRI pulse profiles are $4.4 \sigma$ and $4.7 \sigma$, respectively, and are both more significant than the RXTE-HEXTE profiles for similar energy ranges ( $3.4 \sigma$ and $2.0 \sigma$, respectively) shown in Kuiper et al. (2006). The profile for the $20-50 \mathrm{keV}$ band (Fig. 7E) shows the two peaks separated $\sim 0.5$ in phase. The $50-160 \mathrm{keV}$ profile (Fig. 7F), however, shows only a shoulder to the first pulse at the location of the second pulse (near phase $\sim 0.5$ ) in the $20-50 \mathrm{keV}$ profile. The minimum (DC level) in both cases is located in the same narrow phase interval.

In order to quantify a possible pulse-morphology change as a function of energy, we performed a statistical method based on a combination of a Pearson $\chi^{2}$ (see e.g. Sect. 11.2 of Eadie et al. 1971) and a Run test (see e.g. Sect. 11.3.1 of
Eadie et al. 1971), comparing the shapes of the (independent) 20-50 keV and 50-160 keV pulse profiles. We did not apply the Kolmogorov-Smirnov test, because these background dominated (binned) ISGRI pulse phase distributions have different backgrounds and DC levels. In our approach we first obtained an appropriate model function describing the shape of the $20-50 \mathrm{keV}$ pulse profile by fitting a truncated Fourier series using three harmonics. Next, we fitted the $50-160 \mathrm{keV}$ distribution (in 20 bins) in terms of a constant and a function with a free scale describing the shape of the $20-50 \mathrm{keV}$ pulse profile (d.o.f. $=18$ ). We investigated not only the absolute values of the deviation of the data from the optimum fit, but also the signs of the deviations by combining the probabilities from the independent Pearson $\chi^{2}$ test $\left(P_{\chi^{2}}\right)$ and Run test $\left(P_{\text {run }}\right)$ in an overall joint probability, $P=P_{\chi^{2}} \cdot P_{\text {run }}\left(1-\ln P_{\chi^{2}} \cdot P_{\text {run }}\right)$ (see e.g. Sect. 11.6 of Eadie et al. 1971). In our case the joint probability is 0.104 , thus we cannot claim a significant difference in shape between the $20-50 \mathrm{keV}$ and 50-160 keV ISGRI pulse profiles.

\subsubsection{XMM-Newton and ASCA pulse profiles: time variability}

The ASCA-GIS and RXTE-PCA pulse profiles published by Kuiper et al. (2006) suggest that the pulse profiles below $\sim 2-3 \mathrm{keV}$ are significantly different. However, the RXTE-PCA pulse profiles were averages over years and the RXTE-PCA is not sensitive below $2 \mathrm{keV}$, unlike ASCA-GIS. Dib et al. (2007) used the 10 years of RXTE monitoring above $2 \mathrm{keV}$ to show long-term variability in the pulse-profile shape. However, the reported effect is small; mainly the dip of emission between the two peaks became shallower between 2002 and 2006. Therefore, we used the ASCA-GIS and XMM-Newton observations to create pulse profiles in the same differential energy bands to test for time variability. The differences in the energy resolutions and responses between the two instruments has a negligible impact on the comparison of the profile shapes in the selected broad energy intervals.

Figure 5 shows ASCA-GIS and XMM-Newton pulse profiles in the energy range $0.8-2.0 \mathrm{keV}$. This figure strongly suggests that the pulse profile taken with ASCA-GIS is different from the three XMM-Newton pulse profiles. For ASCA-GIS, the pulse that peaks around phase 0.6 is higher than the pulse that peaks around phase 1.1, while it is the other way around for all three XMM-Newton profiles. The latter profiles seem rather similar. We tested this applying the combination of the Pearson $\chi^{2}$ and Run tests described in Sect. 3.2.1. The XMM-Newton profile with the highest statistics (Obs. B) was used as a template. XMM-Newton profile A, with the lowest statistics, differs from profile $\mathrm{B}$ at the $1.8 \sigma$ level, and profile $\mathrm{C}$ differs from profile $\mathrm{B}$ at the $2.6 \sigma$ level. Slight differences in shape are visible around the peaks of the two pulses, but we do not consider this evidence for significant time variability between the XMM-Newton observations. On the contrary, the ASCA-GIS profile for $0.8-2.0 \mathrm{keV}$ differs from the XMM-Newton profile B at the $16.6 \sigma$ level. The above-mentioned differences in peak heights in the ASCA-GIS and XMM-Newton profiles also become clear when comparing the fluxes in these pulses (see Sect. 3.3.2 and Table 8). The pulse peaking at phase 0.6 contains $\sim 18 \%$ more flux than the average pulse during the XMM-Newton observations. The pulse peaking at phase 1.1 contains $\sim 34 \%$ less flux than the average pulse during the XMM-Newton observations.

Similar conclusions can be reached for the energy band above $2 \mathrm{keV}$ for which the profiles are shown in Fig. 6. XMM-Newton profiles $\mathrm{A}$ and $\mathrm{C}$ differ from profile $\mathrm{B}$ at the 


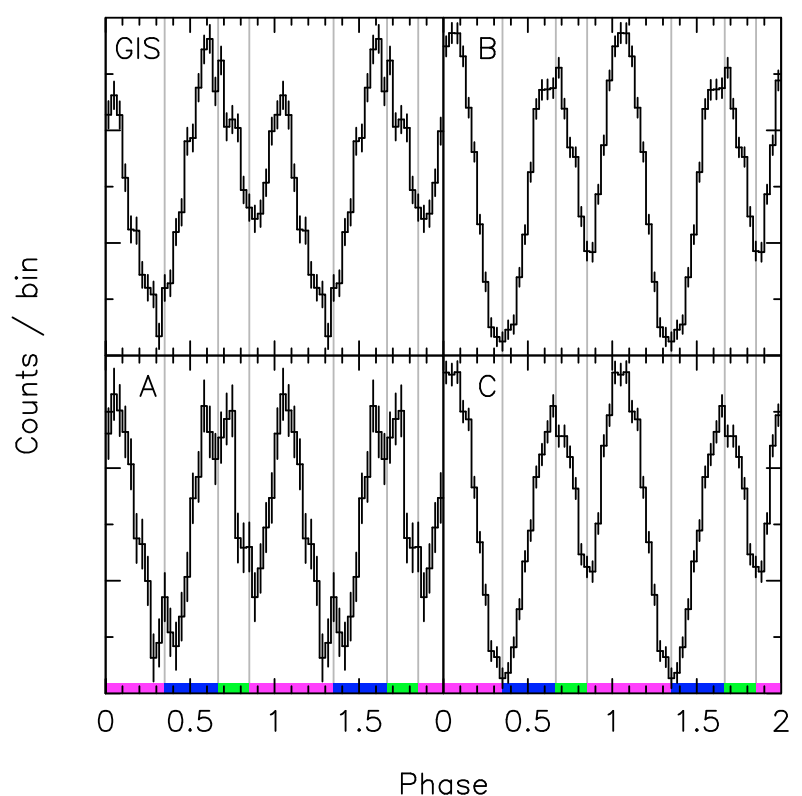

Fig. 5. $4 \mathrm{U} 0142+61$ pulse profiles for $0.8-2.0 \mathrm{keV}$ of ASCA-GIS and the three XMM-Newton observations (A, B, C, Table 3). Phase intervals indicated as in Fig. 4. The profile of XMM-Newton observation $\mathrm{C}$ required a shift in phase by -0.028 for alignment with the profile of observation B (consistent with the other profiles). The relative normalization is consistent with the total pulsed flux $(0.8-2.0 \mathrm{keV})$ being approximately constant (see Table 8 ).

$1.3 \sigma$ and $3.5 \sigma$ levels, respectively ${ }^{3}$; the ASCA-GIS profile for $2.0-8.0 \mathrm{keV}$ deviates at the $9.3 \sigma$ level. The variation is more drastic than the difference in profile shape may indicate. The flux in the GIS pulse peaking at phase 1.1 is $44 \%$ lower than the average flux in the same pulse in the XMM-Newton profiles. The flux in the GIS pulse peaking at phase 0.6 is only $12 \%$ reduced in flux. The difference is evident in Fig. 6 in which the profiles are approximately scaled to the measured $2-8 \mathrm{keV}$ total pulsed flux.

We found significant variability in the overall pulse-profile shapes for energies below $8 \mathrm{keV}$ from the epoch of the ASCA observations (July/August 1999) to the epochs of the XMM-Newton observations in the years 2003-2004 (see Table 3). Particularly in the relative fluxes and spectra of the two pulses the change in shape is more drastic than reported before from RXTE-PCA observations by Dib et al. (2007) and from further XMM-Newton observations by Gonzalez et al. (2008). Morii et al. (2005b) also report pulse-shape changes from the ASCA-GIS data. They use the 1999 observations (which we also use here) as a template to test two shorter observations from 1994 and 1998. Due to the low statistical quality of these data sets only significant changes below $3 \mathrm{keV}$ could be claimed. Dib et al. (2007) notice that the 1998 ASCA-GIS profile presented by Morii et al. (2005b) agrees with the pre-gap RXTE profile while the 1999 one does not. Interestingly, the profile measured with Chandra (Gonzalez et al. 2008) in May 2000 seems to be a transition between the ASCA-GIS (1999) and XMM-Newton profiles. If the deviant ASCA-GIS profile is caused by the glitch prior to this observation as proposed by Morii et al. (2005a), then it took about a year to arrive at the stable situation as measured

\footnotetext{
3 Rea et al. (2007a), analysing the same XMM-Newton observations obtained in 2004, also concluded that there are no significant differences in the profile shapes.
}

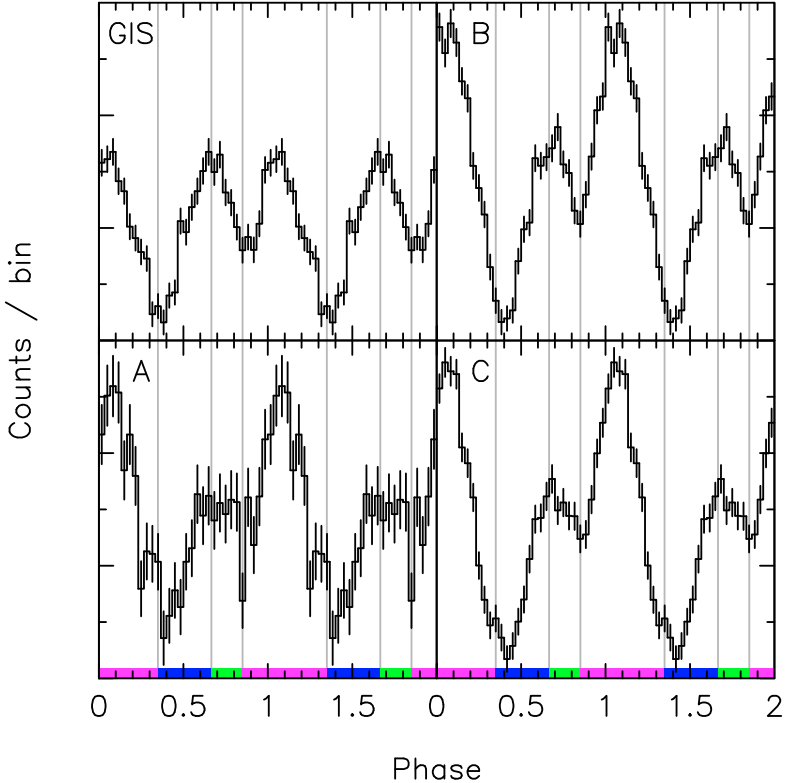

Fig. 6. 4U 0142+61 pulse profiles for $2.0-8.0 \mathrm{keV}$ of ASCA-GIS and the three XMM-Newton observations (A, B, C, Table 3). The relative normalization scales approximately with the total pulsed flux (2-8 keV; see e.g. Table 8). See also the caption of Fig. 5.

with XMM-Newton in 2003-2004 and as can be seen from the RXTE pulse profiles (Dib et al. 2007).

Apparently, some relatively fast reconfiguration in the pulse shape is ongoing during the 1999 ASCA-GIS and 2000 Chandra observations, which might be due to a glitch (Morii et al. 2005b) or other drastic event.

\subsubsection{Pulse-profile changes with energy}

Kuiper et al. (2006) presented pulse profiles as a function of energy from ASCA-GIS, RXTE-PCA and HEXTE. From that work and e.g. Israel et al. (1999); Dib et al. (2007); Rea et al. (2007a) it is clear that the pulse profiles significantly change morphology with energy. In Sect. 3.2.2 we have shown that the pulse profiles of the 2003-2004 XMM-Newton observations are statistically the same. Therefore, we use in this section the summed "time-averaged" pulse profile using all three XMM-Newton observations for comparison with maximal statistics with the profiles derived at higher energies with RXTE-PCA, and INTEGRAL.

In Fig. 7 time-averaged pulse profiles from XMM-Newton, RXTE-PCA, and ISGRI are shown in 6 differential energy bands between $0.8 \mathrm{keV}$ and $160 \mathrm{keV}$. It is evident that the morphology changes are ongoing throughout the energy range. One component that is apparent in all energy bands is the pulse that peaks around phase 1.1 . While the pulse that peaks at phase 0.7 in the XMM-Newton band is vanishing and no longer visible in the RXTE-PCA band, in the INTEGRAL band a pulse is visible around phase 0.5 . In Sect. 3.3 we will disentangle the contributions from different components using phase-resolved spectroscopy.

\subsection{Timing analysis: total-pulsed and phase-resolved pulsed spectra}

Total-pulsed spectra were created by the procedure described in Sect. 2.1.2. An example of how the excess counts in the 


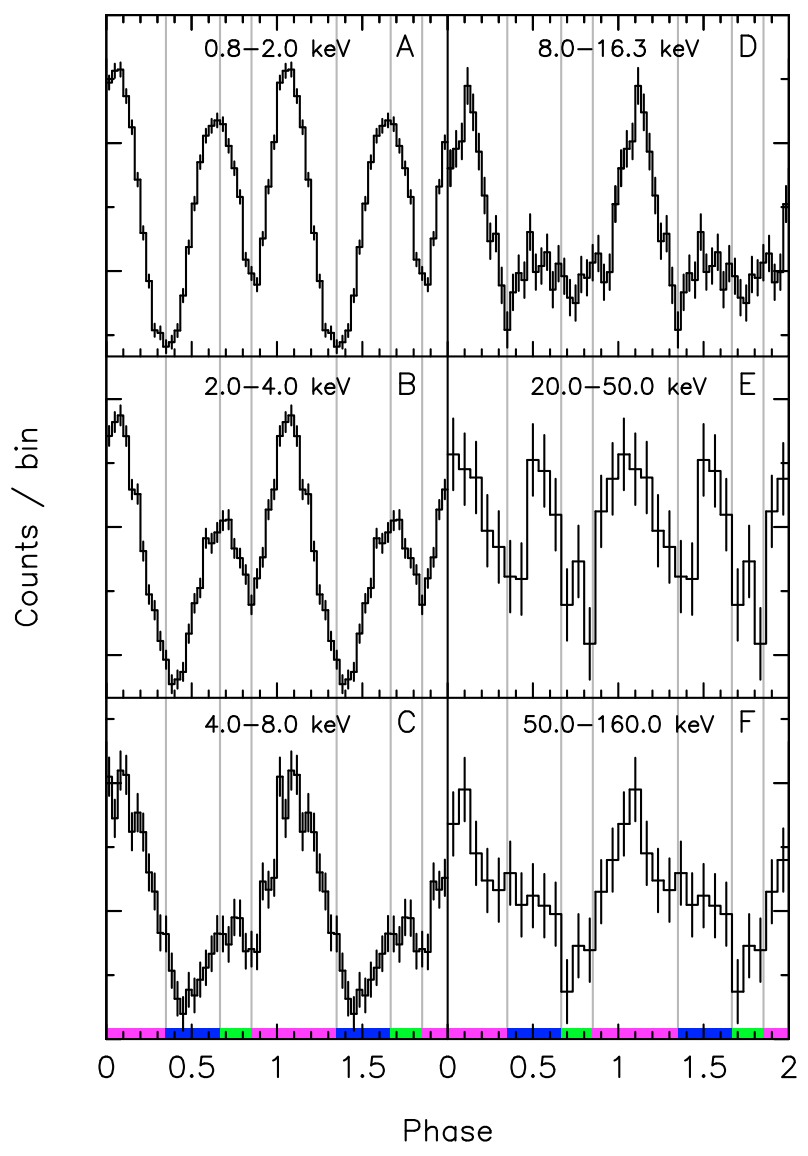

Fig. 7. 4U 0142+61 pulse profiles from soft to hard X-rays. In panels A, $\mathrm{B}$ and $\mathrm{C}$, pulse profiles in the energy ranges $0.8-2.0 \mathrm{keV}, 2.0-4.0 \mathrm{keV}$ and 4.0-8.0 keV are shown which are the sums of the XMM-Newton observations A, B, and C. Panel D presents the RXTE-PCA pulse profile in the energy band 8.0-16.3 keV taken from Kuiper et al. (2006). In panels $\mathrm{E}$ and $\mathrm{F}$ INTEGRAL pulse profiles are shown in the energy ranges $20-50 \mathrm{keV}$ and $50-160 \mathrm{keV}$. Phase intervals are indicated as in Fig. 4.

pulsed components were extracted above a flat background level is shown in Fig. 4.

We performed phase-resolved spectroscopy for the first time for just the pulsed emission. Rea et al. (2007a) also performed phase-resolved spectroscopy with the XMM-Newton data. However, they did not separate the pulsed component from the dominant (on average $\sim 85 \%$ ) DC component, which has a different spectrum and is invariant with phase. For our phase-resolved spectroscopy, we selected three phase intervals for which we want to extract high-energy spectra, using the morphology of the ISGRI 20-160 keV pulse profile (Fig. 4) to define the phase boundaries: $\mathrm{Ph}$ I contains the smaller 2 nd pulse in the INTEGRAL band, Ph II the DC level and Ph III contains the main pulse at hard X-rays, as well as the main pulse for energies below $10 \mathrm{keV}$. The definitions of the phase intervals are given in Table 6 and are indicated with grey vertical lines in all figures with pulse profiles.

\subsubsection{INTEGRAL and RXTE}

For the INTEGRAL data we constructed four broad-band pulse profiles in the energy ranges $20-40 \mathrm{keV}$; $40-80 \mathrm{keV}$; 80-160 keV and 160-300 keV. From these pulse profiles we obtained three total-pulsed flux measurements and one upper limit.
Table 6. Selected phase intervals for extraction of high-energy spectra, using the pulse-shape morphology of the INTEGRAL-IBIS-ISGRI 20-160 keV pulse profile (Fig. 4).

\begin{tabular}{lll}
\hline \hline & Phase interval & Component \\
\hline Ph I & {$[0.35,0.666\rangle$} & Secondary INTEGRAL pulse \\
Ph II & {$[0.666,0.851\rangle$} & DC level INTEGRAL profile \\
Ph III & {$[0.0,0.35\rangle \vee[0.851,1.0\rangle$} & Main INTEGRAL pulse \\
\hline
\end{tabular}

For RXTE-PCA, we extracted 14 pulse profiles in the energy band $2.5-31.5 \mathrm{keV}$ for which we determined the number of excess counts (see Sect. 2.2). The last energy bin (23.8-31.5 keV) yielded no significant pulse profile, but the INTEGRAL and RXTE-PCA spectra nicely bridge the minimum luminosity.

The time-averaged total-pulsed spectra derived from INTEGRAL and RXTE-PCA data are presented in Fig. 8. The spectra are fitted simultaneously using a logparabola for the soft $\mathrm{X}$-rays below $10 \mathrm{keV}$ and either a power law or a logparabola for the hard X-rays. The fit results are summarized in Table 7. In Fig. 8 the fit with two logparabolae is plotted in black while the logparabola and a power-law fit is drawn in grey. Both fits to the INTEGRAL data are fully acceptable, but the single power-law model uses fewer parameters. It can be seen that if the hard X-ray spectrum has a power-law shape with $\Gamma=0.4$ that the pulsed fraction could become as high as $100 \%$ around $\sim 250 \mathrm{keV}$. If the spectral shape is logparabolic, the pulsed fraction would not increase and it would stabilise around $~ 30-40 \%$

For the three differential phase intervals (see Table 6) spectra are also extracted and plotted in Fig. 8. It is clear that Ph III contains the bulk of the energy of the pulsed emission. The spectrum shows a similar transition (from soft to hard) as the totalpulsed spectrum. Also visible is that the soft X-ray spectrum $(<10 \mathrm{keV})$ of $\mathrm{Ph}$ III is harder than the soft X-ray spectra of $\mathrm{Ph} \mathrm{I}$ and Ph II. While below $10 \mathrm{keV} \mathrm{Ph} \mathrm{I} \mathrm{and} \mathrm{Ph} \mathrm{II} \mathrm{form} \mathrm{one} \mathrm{pulse,} \mathrm{in}$ the INTEGRAL band only in Ph I is a pulse visible. From the spectrum and fit in Fig. 8 it can be seen that this transition for $\mathrm{Ph} \mathrm{I}$ is extremely drastic from the very soft to the very hard.

We cannot present an accurate pulsed fraction using RXTEPCA data. The pulsed fraction is defined as the pulsed flux (determined as described in Sect. 2) divided by the total flux from the point source (pulsed $+D C$ ). The RXTE-PCA is a nonimaging instrument, making it difficult to determine a reliable total flux and spectrum due to confusion with RX J0146.9+6121, a closeby high-mass X-ray binary (Motch et al. 1991), and underlying Galactic-ridge emission (Valinia \& Marshall 1998). Alternatively, we present $\eta$ in the bottom panel of Fig. 8. $\eta$ is defined as the fraction of the pulsed emission in a selected phase interval of the total-pulsed emission. From the $\eta$ of $\mathrm{Ph}$ III (magenta) it can be seen that the contribution of this phase interval to the total-pulsed emission increases from $\sim 65 \%$ around $3 \mathrm{keV}$ to practically $100 \%$ around $10 \mathrm{keV}$. The contributions of $\mathrm{PhI}$ and $\mathrm{Ph}$ II gradually decrease from $\sim 20 \%$ at $2 \mathrm{keV}$ to (consistent with) no contribution at $10 \mathrm{keV}$. The contribution of $\mathrm{Ph}$ II remains consistent with $0 \%$ in the INTEGRAL band (DC level), but the contribution to the pulsed emission from $\mathrm{PhI}$ increases again as a result of the small 2nd peak in the INTEGRAL band. Because the pulsed contributions in both phase intervals $\mathrm{Ph} \mathrm{I}$ and $\mathrm{Ph}$ II similarly decrease to $\sim 0 \%$ at $10 \mathrm{keV}$, and the pulse shape in the soft X-ray band seems to be of one component, we infer that the small 2nd pulse in the INTEGRAL band is unrelated to the soft X-ray pulse. It likely originates from a different site (height) in the magnetosphere but appears around the same phase as the soft X-ray pulse. 


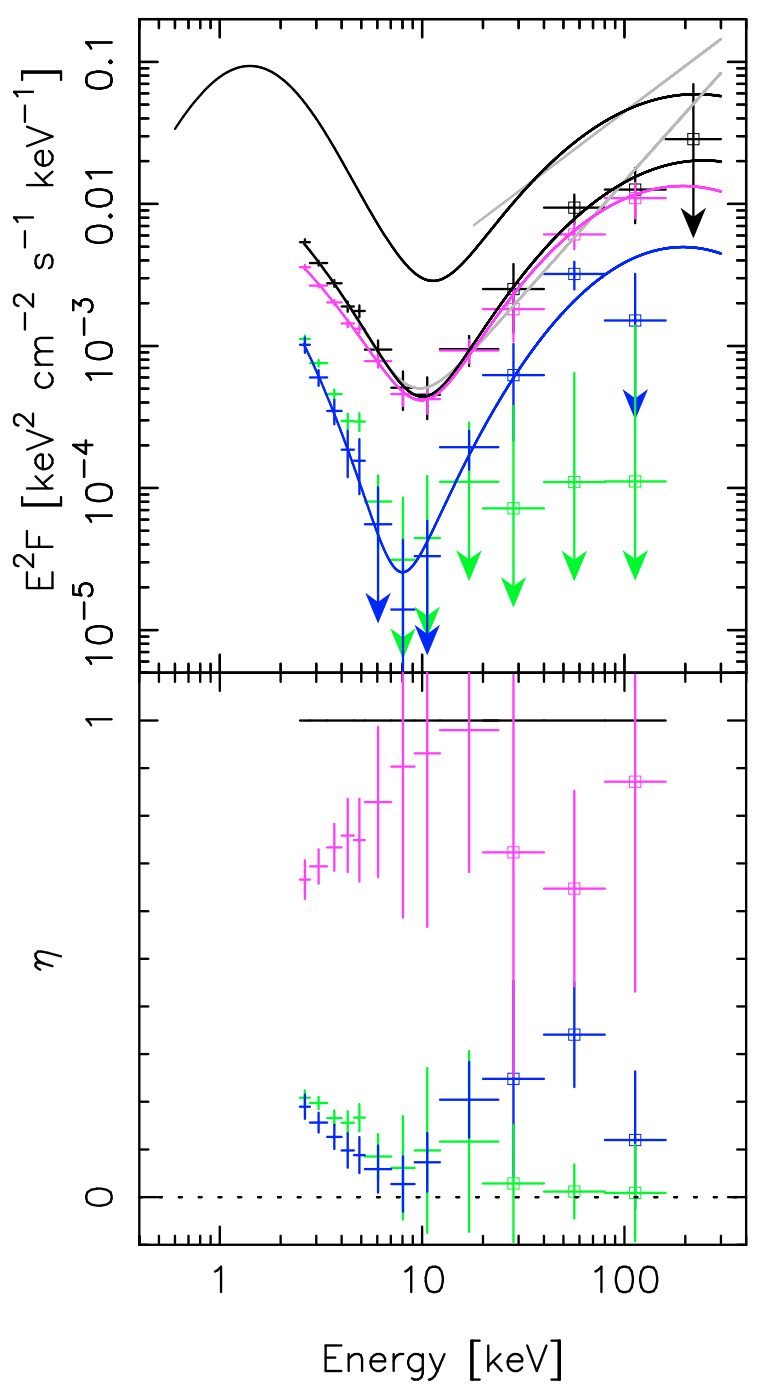

Fig. 8. In the top panel the phase-resolved pulsed-emission spectra and fits of INTEGRAL and RXTE-PCA are presented. Data points with square symbols are INTEGRAL measurements and those without a marker are from RXTE-PCA. Black is the total-pulsed spectrum; in blue $\mathrm{Ph}$ I; in green $\mathrm{Ph}$ II; and in magenta $\mathrm{Ph}$ III. The arrows indicate the flux measurements with a significance less than $1.5 \sigma$. For comparison the INTEGRAL/XMM-Newton-B total-spectrum fit is shown in black. In grey is plotted the power-law fit for the INTEGRAL total-spectrum and the power-law fit for the total-pulsed spectrum. In the bottom panel $\eta$ is presented in the same colour scheme as the spectra. $\eta$ is defined as the fraction of the pulsed emission in a selected phase interval $\mathrm{Ph}$ I, Ph II or $\mathrm{Ph}$ III of the total-pulsed emission, i.e. the sum equals unity.

The time-averaged RXTE-PCA spectra were assembled between March 1996 and September 2003, while the INTEGRAL observations were taken from December 2003 to August 2007. It is then remarkable how well the spectra (total pulsed and $\mathrm{Ph} \mathrm{III)}$ connect in Fig. 8, suggesting stable emission (geometry) above $\sim 10 \mathrm{keV}$ over 11 years.

\subsubsection{XMM-Newton and ASCA}

For the three XMM-Newton and ASCA observations we also determined the total-pulsed spectra as well as the pulsed emission spectra for the three selected phase intervals. Systematic uncertainties in the response for the timing mode of XMM-Newton above $\sim 8 \mathrm{keV}$ prevented us from using the higher-energy data.
Table 7. RXTE-PCA and INTEGRAL spectral-fit parameters for the total-pulsed (TP) spectrum and for three phase intervals (see Table 6). The $N_{\mathrm{H}}$ is fixed to $0.57 \times 10^{22} \mathrm{~cm}^{-2}$. The subscripts below the fit parameters indicate if this parameter accounts for the soft $(s,<10 \mathrm{keV})$ or hard $(h,>10 \mathrm{keV}) \mathrm{X}$-ray part of the spectrum. The normalizations $\left(F_{0 \mathrm{~s}}\right.$ and $\left.F_{0 \mathrm{~h}}\right)$ are taken at $E_{0}=1 \mathrm{keV}$ and have the units $\mathrm{ph} \mathrm{cm}^{-2} \mathrm{~s}^{-1} \mathrm{keV}^{-1}$.

\begin{tabular}{|c|c|c|}
\hline$\overline{\mathrm{TP}}$ & Fit 1 & Fit 2 \\
\hline$\alpha_{\mathrm{s}}$ & $2.27 \pm 1.00$ & $2.82 \pm 1.08$ \\
\hline$\beta_{\mathrm{s}}$ & $1.71 \pm 0.90$ & $1.10 \pm 0.96$ \\
\hline$F_{0 \mathrm{~s}}$ & $(1.34 \pm 0.38) \times 10^{-2}$ & $(1.79 \pm 1.23) \times 10^{-2}$ \\
\hline$\Gamma_{\mathrm{h}}$ & $0.40 \pm 0.15$ & \\
\hline$\alpha_{\mathrm{h}}$ & & $-2.83 \pm 2.16$ \\
\hline$\beta_{\mathrm{h}}$ & & $1.00 \pm 0.67$ \\
\hline$F_{0 \mathrm{~h}}$ & $(9.17 \pm 4.47) \times 10^{-6}$ & $(0.34 \pm 2.62) \times 10^{-8}$ \\
\hline$\chi_{r}^{2}$ (d.o.f.) & 0.78 & $0.50(12)$ \\
\hline$F_{2-10 \mathrm{keV}}\left(\mathrm{erg} \mathrm{cm}^{-2} \mathrm{~s}^{-1}\right)$ & $(6.82 \pm 1.77) \times 10^{-12}$ & $(6.85 \pm 0.93) \times 10^{-12}$ \\
\hline$F_{20-150 \mathrm{keV}}$ & $(2.68 \pm 1.34) \times 10^{-11}$ & $(2.77 \pm 0.68) \times 10^{-11}$ \\
\hline \multicolumn{3}{|l|}{$\mathrm{Ph} \mathrm{I}$} \\
\hline$\alpha_{\mathrm{s}}$ & $2.90 \pm 2.14$ & $2.89 \pm 3.66$ \\
\hline$\beta_{\mathrm{s}}$ & $2.56 \pm 2.88$ & $2.42 \pm 3.35$ \\
\hline$F_{0 \mathrm{~s}}$ & $(0.67 \pm 1.71) \times 10^{-2}$ & $(0.63 \pm 1.74) \times 10^{-2}$ \\
\hline$\Gamma_{\mathrm{h}}$ & $0.12 \pm 0.20$ & \\
\hline$\alpha_{\mathrm{h}}$ & & $-4.00 \pm 4.62$ \\
\hline$\beta_{\mathrm{h}}$ & & $1.31 \pm 0.72$ \\
\hline$F_{0 \mathrm{~h}}$ & $(5.78 \pm 8.81) \times 10^{-7}$ & $(0.67 \pm 5.47) \times 10^{-9}$ \\
\hline$\chi_{r}^{2}$ (d.o.f.) & $1.43(13)$ & $0.69(12)$ \\
\hline$F_{2-10 \mathrm{keV}}\left(\mathrm{erg} \mathrm{cm}^{-2} \mathrm{~s}^{-1}\right)$ & $(1.62 \pm 0.60) \times 10^{-12}$ & $(1.14 \pm 3.64) \times 10^{-12}$ \\
\hline$F_{20-150 \mathrm{keV}}$ & $(5.99 \pm 3.29) \times 10^{-12}$ & $(6.89 \pm 6.42) \times 10^{-11}$ \\
\hline \multicolumn{3}{|l|}{ Ph II } \\
\hline$\alpha_{\mathrm{s}}$ & $2.95 \pm 1.71$ & \\
\hline$\beta_{\mathrm{s}}$ & $1.59 \pm 1.56$ & \\
\hline$F_{0 \mathrm{~s}}$ & $(5.25 \pm 0.72) \times 10^{-3}$ & \\
\hline$\chi_{r}^{2}$ (d.o.f.) & $0.86(15)$ & \\
\hline$F_{2-10 \mathrm{keV}}\left(\mathrm{erg} \mathrm{cm}^{-2} \mathrm{~s}^{-1}\right)$ & $(1.30 \pm 0.03) \times 10^{-12}$ & \\
\hline \multicolumn{3}{|l|}{ Ph III } \\
\hline$\alpha_{\mathrm{s}}$ & $1.63 \pm 0.59$ & $2.68 \pm 0.79$ \\
\hline$\beta_{\mathrm{s}}$ & $2.07 \pm 0.44$ & $1.01 \pm 0.70$ \\
\hline$F_{0 \mathrm{~s}}$ & $(5.55 \pm 2.34) \times 10^{-3}$ & $(1.02 \pm 0.52) \times 10^{-2}$ \\
\hline$\Gamma_{\mathrm{h}}$ & $0.50 \pm 0.11$ & \\
\hline$\alpha_{\mathrm{h}}$ & & $-2.99 \pm 1.73$ \\
\hline$\beta_{\mathrm{h}}$ & & $1.09 \pm 0.54$ \\
\hline$F_{0 \mathrm{~h}}$ & $(1.11 \pm 0.39) \times 10^{-5}$ & $(0.26 \pm 1.67) \times 10^{-7}$ \\
\hline$\chi_{r}^{2}$ (d.o.f.) & $1.1(13)$ & $0.65(12)$ \\
\hline$F_{2-10 \mathrm{keV}}\left(\mathrm{erg} \mathrm{cm}^{-2} \mathrm{~s}^{-1}\right)$ & $(4.72 \pm 0.52) \times 10^{-12}$ & $(4.82 \pm 0.47) \times 10^{-12}$ \\
\hline$F_{20-150 \mathrm{keV}}$ & $(2.06 \pm 0.82) \times 10^{-11}$ & $(2.07 \pm 1.09) \times 10^{-11}$ \\
\hline
\end{tabular}

For this reason the observational gap between XMM-Newton and INTEGRAL is about $12 \mathrm{keV}$.

In the top panels of Fig. 9 the results of ASCA-GIS and XMM-Newton obs. A, B and C are presented together with the INTEGRAL results of Fig. 8. The colour scheme is the same as in Fig. 8. In black are drawn the total-pulsed spectra and (except for ASCA-GIS) the (binned) total spectra. The best fits are drawn to the total spectra of XMM-Newton and INTEGRAL. In the panel for ASCA-GIS the fit to the total spectrum for XMM-Newton obs. C and INTEGRAL is drawn.

We have fitted each spectrum with a single logparabolic function. These fit results are given in Table 8. The parameters of a logparabolic function are not easy to compare, but the flux values indicate that there are some differences between the different observations, consistent with our findings in the comparison of the pulse shapes.

Most remarkable are flux differences between the ASCAGIS observation and the XMM-Newton ones. (The latter are consistent with the fluxes reported by Gonzalez et al. 2008). While 

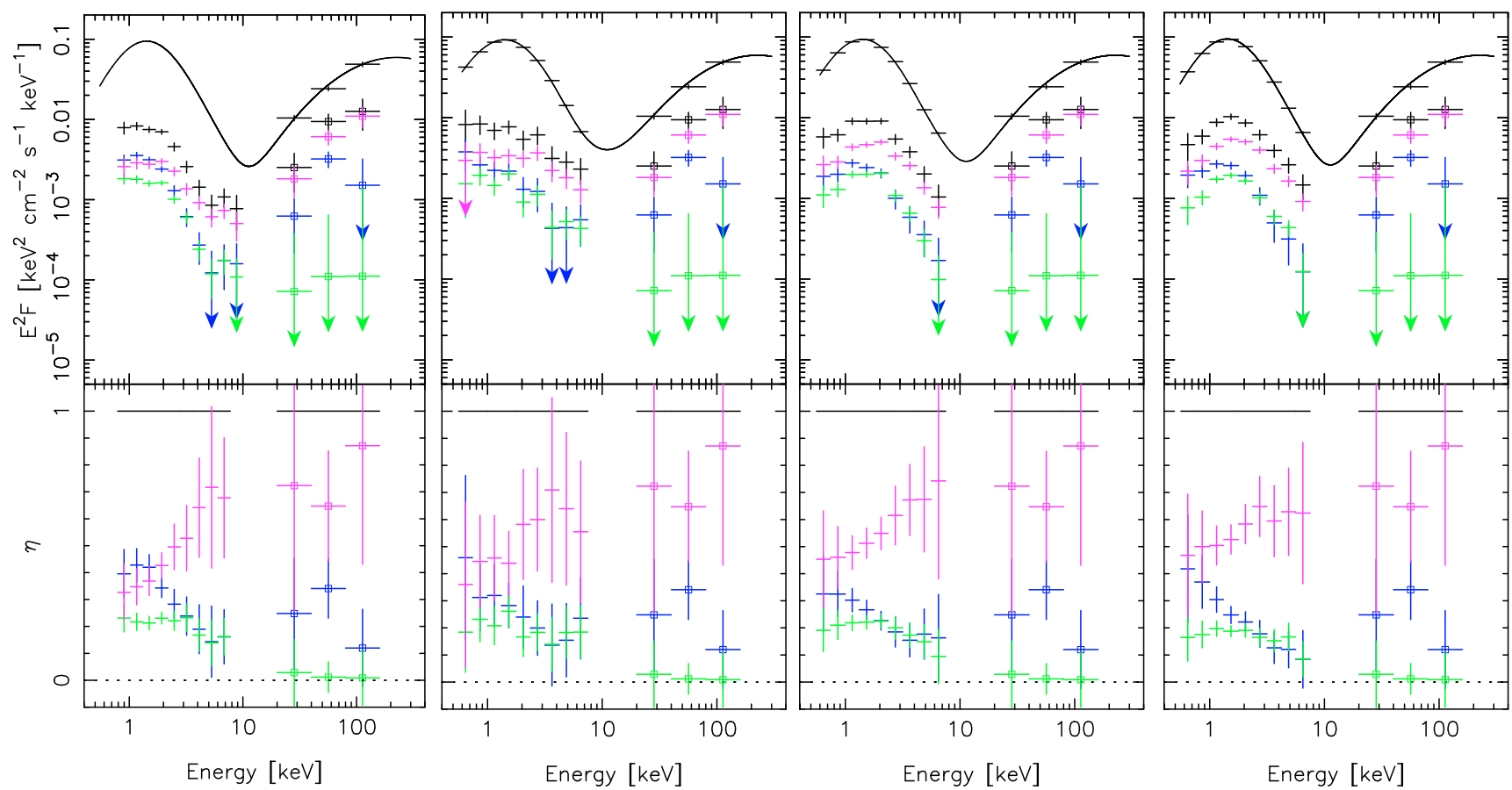

Fig. 9. For four (soft) X-ray and INTEGRAL observations shown are the total, total-pulsed and phase-resolved pulsed spectra (top panels) and $\eta$ (bottom panels, defined as the fraction of the total pulsed emission). The (soft) X-ray data from left to right are ASCA GIS, XMM-Newton PN obs. A, B and C. All data are equally binned for comparison. For the total spectra, the best fit is shown. In the top panel for ASCA-GIS the best fit to the XMM-Newton-B-INTEGRAL total spectrum is shown for clarity. The total spectra and total-pulsed spectra are plotted in black. The phase resolved spectra for Ph I, Ph II and Ph III (see Table 6) are plotted in blue, green and magenta, respectively. The INTEGRAL phase-resolved spectra are indicated in each figure with a square symbol. The data points with arrows indicate that the flux values have significances less than $1.5 \sigma$. The positive $1 \sigma$ error is drawn. In the bottom panels $\eta$ is presented in the same colour scheme as the spectra.

Table 8. ASCA-GIS and XMM-Newton (A, B and C) fits for the total-pulsed (TP) spectra and for spectra of three phase intervals (Ph I, II and III, see Table 6) for $4 \mathrm{U} 0142+61$. The $N_{\mathrm{H}}$ is fixed to $0.57 \times 10^{22} \mathrm{~cm}^{-2}$. For each observation the three parameters of the parabola are given. The normalizations $\left(F_{0}\right)$ are taken at $E_{0}=1 \mathrm{keV}$ and have the units $\mathrm{ph}^{-2} \mathrm{~s}^{-1} \mathrm{keV}^{-1}$.

\begin{tabular}{|c|c|c|c|c|}
\hline TP & ASCA-GIS & A & B & $\overline{\mathrm{C}}$ \\
\hline$\alpha$ & $1.88 \pm 0.30$ & $2.19 \pm 0.38$ & $1.24 \pm 0.15$ & $1.12 \pm 0.17$ \\
\hline$\beta$ & $2.03 \pm 0.48$ & $0.65 \pm 0.55$ & $2.43 \pm 0.26$ & $2.41 \pm 0.27$ \\
\hline$F_{0}$ & $(8.49 \pm 0.77) \times 10^{-3}$ & $(7.93 \pm 0.10) \times 10^{-3}$ & $(8.17 \pm 0.41) \times 10^{-3}$ & $(7.98 \pm 0.45) \times 10^{-3}$ \\
\hline$\chi_{r}^{2}$ (d.o.f.) & $1.8(7)$ & $0.29(6)$ & $0.88(15)$ & $0.86(15)$ \\
\hline$F_{0.8-2 \mathrm{keV}}\left(\mathrm{erg} \mathrm{cm}^{-2} \mathrm{~s}^{-1}\right)$ & $(11.47 \pm 0.70) \times 10^{-12}$ & $(10.96 \pm 1.38) \times 10^{-12}$ & $(12.64 \pm 0.50) \times 10^{-12}$ & $(12.72 \pm 0.56) \times 10^{-12}$ \\
\hline$F_{2-10 \mathrm{keV}}\left(\mathrm{erg} \mathrm{cm}^{-2} \mathrm{~s}^{-1}\right)$ & $(5.55 \pm 0.33) \times 10^{-12}$ & $(9.02 \pm 1.20) \times 10^{-12}$ & $(8.16 \pm 0.35) \times 10^{-12}$ & $(9.17 \pm 0.39) \times 10^{-12}$ \\
\hline \multicolumn{5}{|l|}{$\mathrm{Ph} \mathrm{I}$} \\
\hline$\alpha$ & $1.54 \pm 0.27$ & $2.72 \pm 0.47$ & $1.57 \pm 0.20$ & $1.59 \pm 0.20$ \\
\hline$\beta$ & $3.76 \pm 0.68$ & $0.39 \pm 0.84$ & $2.71 \pm 0.45$ & $2.79 \pm 0.45$ \\
\hline$F_{0}$ & $(3.49 \pm 0.28) \times 10^{-3}$ & $(2.57 \pm 0.38) \times 10^{-3}$ & $(2.53 \pm 0.14) \times 10^{-3}$ & $(2.57 \pm 0.14) \times 10^{-3}$ \\
\hline$\chi_{r}^{2}$ (d.o.f.) & $0.66(7)$ & $0.30(6)$ & $0.61(15)$ & $0.50(15)$ \\
\hline$F_{0.8-2 \mathrm{keV}}\left(\mathrm{erg} \mathrm{cm}^{-2} \mathrm{~s}^{-1}\right)$ & $(4.58 \pm 0.46) \times 10^{-12}$ & $(3.29 \pm 0.80) \times 10^{-12}$ & $(3.56 \pm 0.27) \times 10^{-12}$ & $(3.37 \pm 0.25) \times 10^{-12}$ \\
\hline$F_{2-10 \mathrm{keV}}\left(\mathrm{erg} \mathrm{cm}^{-2} \mathrm{~s}^{-1}\right)$ & $(1.39 \pm 0.14) \times 10^{-12}$ & $(1.83 \pm 0.52) \times 10^{-12}$ & $(1.50 \pm 0.16) \times 10^{-12}$ & $(1.36 \pm 0.12) \times 10^{-12}$ \\
\hline \multicolumn{5}{|l|}{ Ph II } \\
\hline$\alpha$ & $1.51=$ & $2.24 \pm 0.39$ & $0.94 \pm 0.18$ & $0.97 \pm 0.17$ \\
\hline$\beta$ & $2.81=$ & $0.83 \pm 0.63$ & $3.27 \pm 0.34$ & $2.97 \pm 0.31$ \\
\hline$F_{0}$ & $(1.79 \pm 0.16) \times 10^{-3}$ & $(1.80 \pm 0.23) \times 10^{-3}$ & $(1.75 \pm 0.10) \times 10^{-3}$ & $(1.51 \pm 0.08) \times 10^{-3}$ \\
\hline$\chi_{r}^{2}$ (d.o.f.) & $1.73(7)$ & $1.09(6)$ & $0.96(15)$ & $1.35(15)$ \\
\hline$F_{0.8-2 \mathrm{keV}}\left(\mathrm{erg} \mathrm{cm}^{-2} \mathrm{~s}^{-1}\right)$ & $(2.50 \pm 0.26) \times 10^{-12}$ & $(2.44 \pm 0.50) \times 10^{-12}$ & $(2.77 \pm 0.20) \times 10^{-12}$ & $(2.41 \pm 0.18) \times 10^{-12}$ \\
\hline$F_{2-10 \mathrm{keV}}\left(\mathrm{erg} \mathrm{cm}^{-2} \mathrm{~s}^{-1}\right)$ & $\times 10^{-12}$ & $\pm 0.39) \times 1($ & $1 \pm 0.10) \times 1$ & $8 \pm 0.10) \times 10^{-12}$ \\
\hline \multicolumn{5}{|l|}{ Ph III } \\
\hline$\alpha$ & 1.87 & $1.74 \pm 0$ & $1.05 \pm 0.14$ & $0.95 \pm 0.14$ \\
\hline$\beta$ & $1.36 \pm 0.44$ & $0.95 \pm 0.55$ & $2.31 \pm 0.21$ & $2.41 \pm 0.20$ \\
\hline$F_{0}$ & $(3.03 \pm 0.37) \times 10^{-3}$ & $(3.42 \pm 0.55) \times 10^{-3}$ & $(3.86 \pm 0.19) \times 10^{-3}$ & $(3.98 \pm 0.19) \times 10^{-3}$ \\
\hline$\chi_{r}^{2}$ (d.o.f.) & $1.72(7)$ & $0.27(6)$ & $1.01(15)$ & $1.54(15)$ \\
\hline$F_{0.8-2 \mathrm{keV}}\left(\mathrm{erg} \mathrm{cm}^{-2} \mathrm{~s}^{-1}\right)$ & $(4.23 \pm 0.58) \times 10^{-12}$ & $(5.09 \pm 1.17) \times 10^{-12}$ & $(6.30 \pm 0.37) \times 10^{-12}$ & $(6.65 \pm 0.36) \times 10^{-12}$ \\
\hline$F_{2-10 \mathrm{keV}}\left(\mathrm{erg} \mathrm{cm}^{-2} \mathrm{~s}^{-1}\right)$ & $(3.01 \pm 0.28) \times 10^{-12}$ & $(5.37 \pm 0.82) \times 10^{-12}$ & $(5.13 \pm 0.24) \times 10^{-12}$ & $(5.63 \pm 0.22) \times 10^{-12}$ \\
\hline
\end{tabular}




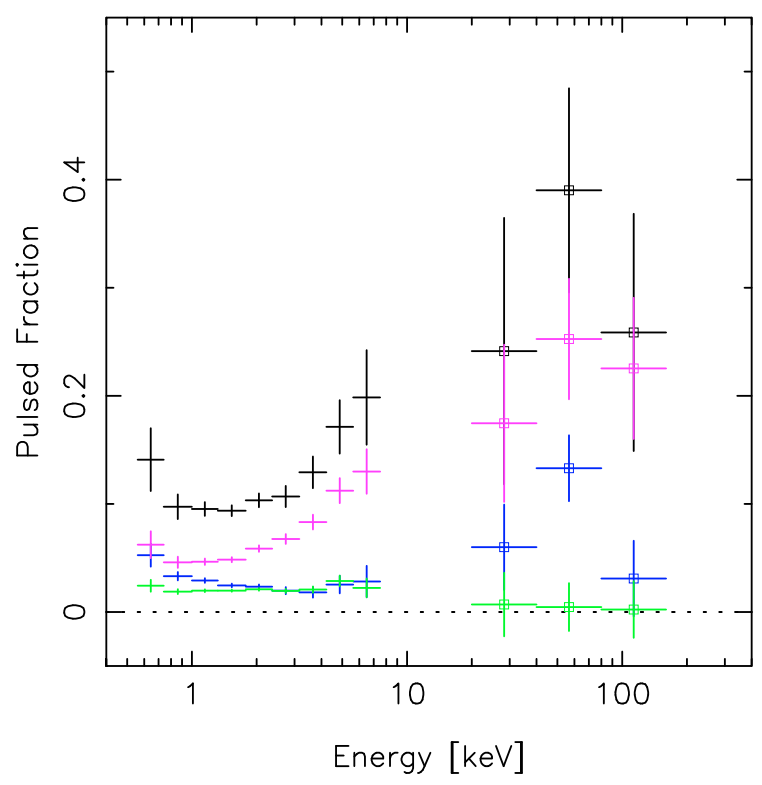

Fig. 10. Pulsed fraction as function of energy. Below $10 \mathrm{keV}$ the averaged XMM-Newton pulsed fraction of observations A, B and C are plotted. Above $10 \mathrm{keV}$ the INTEGRAL pulsed fraction is plotted. Colour coding as in Figs. 8 and 9; Total-pulsed emission (black), $\mathrm{Ph}$ I (blue), $\mathrm{Ph}$ II (green), Ph III (magenta).

the $0.8-2 \mathrm{keV}$ total-pulsed fluxes of all observations are comparable, the $2-10 \mathrm{keV}$ total-pulsed flux of ASCA-GIS is significantly lower than the rest. From the phase-resolved spectra it becomes clear that this difference is due to the significantly lower flux $(2-10 \mathrm{keV})$ in the main pulse (Ph III). In this phase interval the ASCA-GIS 2-10 keV flux is less than in the XMM-Newton observations, while the $2-10 \mathrm{keV}$ flux for phases $\mathrm{Ph} \mathrm{I}$ and $\mathrm{Ph}$ II are slightly lower than, or comparable to those measured with XMM-Newton. We note that the $0.8-2 \mathrm{keV}$ ASCA-GIS flux in $\mathrm{Ph}$ III is somewhat lower than in the other observations. Note also that only for ASCA-GIS the $0.8-2 \mathrm{keV}$ flux in $\mathrm{Ph} \mathrm{I}$ is higher than, or comparable to, this flux in $\mathrm{Ph}$ III.

Table 8 shows that over the three XMM-Newton observations the total-pulsed flux in the $0.8-2 \mathrm{keV}$ and $2-8 \mathrm{keV}$ bands as well as the fluxes in all three phase intervals are statistically stable. The latter is consistent with the conclusion that the shape of the pulse profiles did not change measurably in this time interval.

\subsubsection{Comparison}

To determine the pulsed fraction as a function of energy we have binned the total spectra in the same binning as the total-pulsed and phase-resolved pulsed spectra. The pulsed fraction is defined as the pulsed flux (determined as described in Sect. 2) divided by the total flux from the point source (pulsed + DC). For the instruments ASCA-GIS and RXTE-PCA we do not have accurate total spectra, therefore we also consider $\eta$.

We start with the pulsed fraction. In the previous sections we showed that XMM-Newton observations A, B and C exhibit statistically identical pulse shapes and pulsed fluxes. Therefore, we have combined these observations by taking weighted averages. In Fig. 10 the pulsed fraction as a function of energy is presented for the averaged XMM-Newton and INTEGRAL observations (black data points). Also presented in this figure are the pulsed fractions for the three selected phase intervals (coloured data points). It can be seen that the pulsed fraction in the XMM-Newton band increases from $\sim 10 \%$ around $1 \mathrm{keV}$

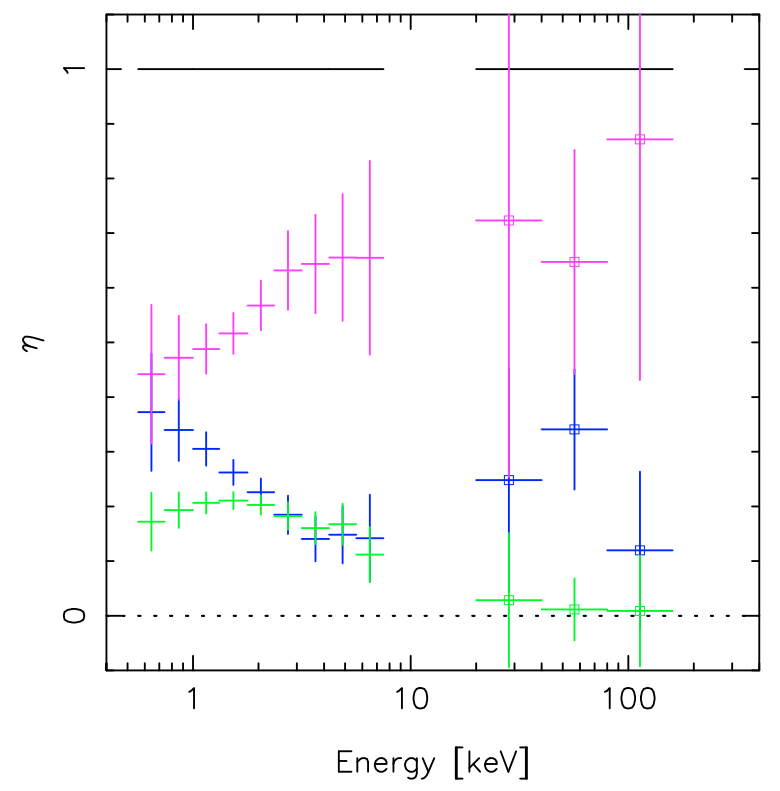

Fig. 11. Contribution to the total-pulsed emission $\eta$ of the three selected phase intervals from the averaged XMM-Newton $\eta$ of observations A, $\mathrm{B}$ and $\mathrm{C}$ and for INTEGRAL (>10 keV). Colour coding as in Fig. 10.

to $\sim 20 \%$ around $8 \mathrm{keV}$. This trend continues in the INTEGRAL band where the pulsed fraction increases to possibly as high as $\sim 40 \%$ in the $40-80 \mathrm{keV}$ band. Rea et al. (2007a) presented the pulsed fraction of the fundamental and first harmonic sine functions for the XMM-Newton pulse profiles as a function of energy below $10 \mathrm{keV}$. These are consistent with the total pulsed fractions we show in Fig. 10.

Figure 10 also shows that the trend set by the total-pulsed fraction is followed closely by the pulsed fraction that can be assigned to Ph III (magenta), as we have already discussed in Sect. 3.3.1. Ph III alone accounts for $\sim 5-12 \%$ of the pulsed fraction between $1-8 \mathrm{keV}$. The other two phases do not show the same trend. $\mathrm{PhI}$, the 2nd pulse in the INTEGRAL band, accounts for $\sim 5 \%$ at $1 \mathrm{keV}$ decreasing to $\sim 3 \%$ at $8 \mathrm{keV}$. Finally, $\mathrm{Ph}$ II is almost constant in this energy range at a level of $\sim 2.5 \%$. These trends are also visible in the phase-resolved pulsed spectra in Fig. 9, showing that at higher energies around $8 \mathrm{keV} \mathrm{Ph}$ III dominates over the other two phase intervals.

To investigate this further, the distribution of $\eta$ is even more illustrative, as can be seen in Figs. 9 and 11: the contribution of $\mathrm{Ph}$ III to the total pulsed emission steadily increases with energy, reaching a fraction of $\sim 65 \%$ at $8 \mathrm{keV}$. For the avarage of the above reported RXTE-PCA observations (Fig. 8) the contribution from $\mathrm{Ph}$ III increases even to $\sim 100 \%$ around $10 \mathrm{keV}$. In the INTEGRAL band this contribution is again down to $\sim 60-70 \%$, due to the new pulse in $\mathrm{Ph}$ I.

In Fig. 9 it is also clearly visible that during the ASCAGIS observation the $\eta$ of Ph III below $2 \mathrm{keV}$ is less than that of $\mathrm{Ph}$ I. In all observations the $\eta$ of $\mathrm{Ph}$ I decreases with energy from $\sim 30-40 \%$ to $\$ 5 \%$ around $8 \mathrm{keV}$. However, above $10 \mathrm{keV}$ the contribution of $\mathrm{Ph}$ I increases again due to the 2 nd pulse in the INTEGRAL band. Ph II, which was chosen as the DC level of unpulsed emission in the INTEGRAL band, first shows a constant $\eta$ of $\sim 20 \%$ and it decreases from $2 \mathrm{keV}$ gradually to $0 \%$ in the INTEGRAL band. 


\section{Summary}

In this paper we have presented new and more detailed characteristics of $4 \mathrm{U} 0142+61$ in the hard X-ray regime $(>10 \mathrm{keV})$ and studied these in a broader high-energy view using archival soft X-ray data $(<10 \mathrm{keV})$ in consistent analyses. Many new or more accurate results are reported which should be considered in theoretical modelling of the high-energy emission from AXPs. Particularly new for studies of AXPs is the performed phase resolved spectroscopy over the total high-energy band, revealing distinctly different components contributing to the total emission. We will first present a summary of the results.

\subsection{Total high-energy emission of $4 U 0142+61$}

1) With an effective exposure of $2.4 \mathrm{Ms}$ ISGRI measures the most accurate time-averaged total spectrum between 20 and $230 \mathrm{keV}$ which is fully consistent with a power-law shape with index $0.93 \pm 0.06$ (Sect. 3.1.2, Fig. 3 and Table 4).

2) Combining ISGRI and SPI spectral data provides the first evidence at the $\sim 4 \sigma$ level for a spectral break with maximum luminosity at an energy of $\sim 270 \mathrm{keV}$, assuming a logparabolic shape of the hard X-ray spectrum (Sect. 3.1.2 and Fig. 3).

3) The total high-energy spectrum, combining spectra measured with XMM-Newton and ISGRI, can be described satisfactorily with the sum of three logparabolic functions, two dominating the spectrum for energies below $8 \mathrm{keV}$ and one above $20 \mathrm{keV}$ (see also Rea et al. 2007b). Minimum values for the luminosity are reached around $10 \mathrm{keV}$ (Sect. 3.1.4, Fig. 3 and Table 5).

\subsection{Long-term variability in the total emission of $4 U 0142+61$}

1) ISGRI does not reveal evidence for significant long-term variability of the source flux and power-law spectral index on one-year time scales between December 2003 and August 2006 at the $17 \%(1 \sigma)$ level (Sect. 3.1.1, Figs. 1 and 2 and Table 4).

2) Two short INTEGRAL ToO observations following bursting behaviour of $4 \mathrm{U} 0142+61$ measured with RXTE show the AXP to be in a state consistent with the time averaged flux and spectral shape. The total flux was stable within $\sim 20 \%$ $(1 \sigma ;$ Sect. 3.1.3).

\subsection{Pulse profiles}

1) Above $20 \mathrm{keV}$ ISGRI measures significant hard-X-ray pulse profiles to $160 \mathrm{keV}$ (Sect. 3.2.1 and Fig. 4).

2) The morphologies of the profiles measured below $10 \mathrm{keV}$ with XMM-Newton and ASCA-GIS differ from the hard$\mathrm{X}$-ray profile; one of the two X-ray pulses vanishes around $10 \mathrm{keV}$ (confirmed with RXTE-PCA) and a new pulse appears above $20 \mathrm{keV}$ (Sect. 3.2.3 and Fig. 7).

3) Three XMM-Newton observations in 2003-2004 give statistically identical pulse profiles, but the profile measured by ASCA-GIS in 1999 following a possible glitch of 4U 0142+61 (see also Morii et al. 2005b; Dib et al. 2007) is significantly different with different relative strengths and spectra $(0.8-10 \mathrm{keV})$ of the two pulses visible in the pulse profiles below $10 \mathrm{keV}$ (Sect. 3.2.2 and Figs. 5 and 6).

\subsection{Pulsed spectra}

1) ISGRI measures above $20 \mathrm{keV}$ a total-pulsed spectrum up to $160 \mathrm{keV}$ with a shape consistent with a power law with index $0.40 \pm 0.15$, significantly different from that of the total spectrum. This implies that the difference spectrum, the DC component, is a genuinely different component (Sect. 3.3.1, Fig. 8 and Table 7).

2) Below $10 \mathrm{keV}$, there is evidence of long-term time variability. The total pulsed fluxes measured for the $0.8-2.0 \mathrm{keV}$ band with ASCA-GIS in 1999 and in the three XMM-Newton observations in 2003-2004 are comparable, but in the $2.0-10.0 \mathrm{keV}$ band different flux values are measured. The ASCA-GIS value is significantly lower by $\sim 35 \%$ than the mutually consistent XMM-Newton values, and the RXTEPCA flux appears to be in between. The $\sim 35 \%$ decrease in total pulsed flux in the ASCA-GIS observation can largely be assigned to a decrease in the strength of only one of the X-ray pulses (Sect. 3.3, Fig. 9 and Tables 7 and 8). See also Dib et al. (2007) for a significant increased pulsed flux of $~ 36 \%$ since 2004 and during the bursting activity in 2006-2007 (Gonzalez et al. 2008).

3) The total pulsed fraction increases from $\sim 10 \%$ at $1 \mathrm{keV}$ to possibly as high as $\sim 40 \%$ in the $40-80 \mathrm{keV}$ band. Above these energies this fraction is uncertain, but might become close to $100 \%$, depending on the actual shapes of the total and pulsed spectra (Sect. 3.3.3 and Fig. 10).

\subsection{Phase-resolved pulsed spectra}

1) Phase-resolved spectroscopy over the total high-energy band reveals at least three genuinely different pulse components with different spectra: a) a component in phase interval $\mathrm{Ph}$ III (phase $0.85-1.35$ ) peaking at phase 1.1 , present and dominant from $0.8 \mathrm{keV}$ to $160 \mathrm{keV}$; b) a hard-X-ray component in phase interval $\mathrm{Ph}$ I peaking in the ISGRI band above $20 \mathrm{keV}$ at phase $\sim 0.55$; c) a soft X-ray component in phase interval $\mathrm{Ph} \mathrm{I}+\mathrm{Ph}$ II (phase 0.35-0.85) peaking in the profiles for energies below $10 \mathrm{keV}$ at phase $\sim 0.65$, and not visible above $10 \mathrm{keV}$; d) the soft X-ray component mentioned in c) appears to consist of two parts, a softer contribution from phase interval $\mathrm{Ph}$ II than from phase interval $\mathrm{Ph}$ I, consistently seen for all reported X-ray observations (Sect. 3.3, Fig. 9 and Tables 7 and 8).

2) The total pulsed spectra and that of $\mathrm{Ph}$ III measured with RXTE (1996-2003) and consecutively with ISGRI (2003-2007) connect smoothly, and the phase-resolved spectra measured with ASCA (1999), XMM-Newton (2003-2004) and RXTE (1996-2003) are similar. This is indicative of a remarkably stable geometry of the contributing emission sites at the surface and/or in the magnetosphere of the magnetar. There is no evidence of a drastic reordering (Sect. 3.3.1, Fig. 8 and Table 7).

\section{Discussion}

In this section, we discuss how these new findings fit within the theoretical models attempting to explain hard X-ray emission from AXPs. Before the discovery by INTEGRAL of nonthermal emission from AXPs above $10 \mathrm{keV}$, Cheng \& Zhang (2001) modeled the production of high-energy gamma radiation in vacuum gaps proposed to be present in the outer magnetospheres of AXPs. Gamma-ray emission at the polar caps will be quenched due to the strong field, but far away from the 
pulsar surface gamma radiation could be emitted because the local field will drop below the critical quantum limit. This radiopulsar scenario could offer a stable configuration, suggested by our results to be required, but their model calculations do not reproduce the hard spectra and high X-ray luminosities found for e.g. $4 U 0142+61$. After the discovery by INTEGRAL three approaches were explored specifically to address the non-thermal, very luminous hard X-ray emission from AXPs: 1) a quantum electrodynamics model by Heyl \& Hernquist (2005a,b); 2) a corona model by Beloborodov \& Thompson (2007) and most recently by Lyubarsky \& Eichler (2008); 3) a resonant upscattering model by Baring \& Harding (2007). Below, we will consider each of these attempts.

\subsection{Quantum electrodynamics model}

Heyl \& Hernquist (2005a) created a model to explain the origin of the SGR and AXP bursts through fast Quantum Electrodynamics (QED) wave modes which could be triggered when a starquake or other rearrangement in the magnetic field occurs. In their fast-mode break down model, magnetohydrodynamic (MHD) waves generated near the magnetar surface propagating outwards through the magnetosphere are altered by vacuum polarization. Hydrodynamic shocks can develop and electron-positron pairs produced. An optically thick fireball could be created emitting mainly $X$ and gamma rays, which can be observed as a SGR or an AXP burst. To explain stable non-thermal emission Heyl \& Hernquist (2005b) extended this model to weaker fast modes that do not create an optically thick fireball. The resulting spectrum depends on the total energy delivered by the fast mode and the maximum magnetic field strength. The latter value determines two energy values where the spectrum breaks. The peak energy in the spectrum is given by $E_{\text {break }} \approx 1600 \frac{B_{\text {crit }}}{B} m c^{2}$, where $B_{\text {crit }}$ is the quantum critical field of $4.4 \times 10^{13} \mathrm{G}$, and $B$ the neutron-star magnetic field. The other break energy is given by $E_{0} \approx 10^{-6} \frac{B}{B_{\text {crit }}} m c^{2}$. The spectral shapes below, between and above these break energies are power-law like with photon indices of $0,1.5$ and 3 , respectively. The power law below $E_{0}$ can extend down to the optical regime, in which pulsed non-thermal optical emission (Eichler et al. 2002) has been detected from 4U 0142+61 (Hulleman et al. 2004; Kern \& Martin 2002; Dhillon et al. 2005).

Peak energies predicted by this model all exceed $1 \mathrm{MeV}$. The fast mode cascade of pairs is not effective if the energy in the pairs $\left(E_{\text {break }}\right)$ is less than $2 m c^{2}$. This natural limit is called the minimal model where $E_{\mathrm{break}} \approx 1 \mathrm{MeV}$. In this case, the pairs are created in a (local) magnetic field much weaker than $B_{\text {crit }}$. Heyl (2007) examined the angular dependence of the nonthermal emission in the weak field regime ( $\left.B_{\mathrm{NS}} \ll B_{\text {crit }}\right)$. The produced spectrum strongly depends on the angle between the magnetic field and the wave propagation direction. Specifically, the non-thermal hard X-ray emission is expected to be largest when the observer's viewing direction to the pulsar is perpendicular to the local magnetic field, while the thermal emission is expected to peak when the observer views the polar regions. The difference between the phases at which the thermal and nonthermal emissions peak can range between $90^{\circ}$ and $180^{\circ}$ if the angles $\alpha+\beta \geq 90^{\circ}$, where $\alpha$ is the angle between the rotation axis and the observer's line of sight and $\beta$ the angle between the rotation axis and the magnetic (dipole) axis. If $\alpha+\beta<90^{\circ}$, then the phase difference between the non-thermal and the thermal emission is $180^{\circ}$.
Comparing the above QED model predictions with our results, we note that the power-law spectral index of the total emission above $20 \mathrm{keV}$ of $4 \mathrm{U} 0142+61,0.93 \pm 0.06$, is harder than the predicted value of 1.5. Furthermore, the estimated break energy of $\sim 270 \mathrm{keV}$ is far below the allowed minimum value of $\sim 1 \mathrm{MeV}$, and would mean a neutron star magnetic field strength of $1.3 \times 10^{16} \mathrm{G}$. The spectral index of the total pulsed emission of $0.40 \pm 0.15$ is inconsistent with the value 1.5 . We note that Heyl \& Hernquist (2005a) do not make a distinction between the pulsed and total emission.

Finally, there is a clear prediction of a phase difference between the thermal and non-thermal emission: our phase-resolved spectroscopy shows that the actual situation is much more complex: one pulse exhibits a spectrum composed of a thermal and a non-thermal component in the same phase interval (Ph III); the thermal pulse in phase interval $\mathrm{Ph} \mathrm{I}+\mathrm{II}$ seems to vanish above $10 \mathrm{keV}$ and a non-thermal pulse shows up above $20 \mathrm{keV}$ in $\mathrm{Ph} \mathrm{II}$, also at a negligible phase difference.

\subsection{Corona model}

The corona model developed by Beloborodov \& Thompson (2007) is an extension of earlier work by Thompson \& Beloborodov (2005) and Thompson et al. (2002). Thompson et al. (2002) extended the magnetar model by a twisted magnetosphere, which was introduced by Thompson et al. (2000) to explain some observational characteristics seen in SGR 1900+14. Accordingly, the assumed dipolar field becomes twisted due to continuous seismic activity as a result of the extreme internal magnetic field and a quasi stable loop (tube) can be formed with a maximum radius of $\sim 2 R_{\mathrm{NS}}$.

Essential in this scenario is an electric field parallel to the magnetic field which is generated by self induction. This parallel electric field is capable of accelerating particles and initiates an electron-positron avalanche. In this way, toroidal field (magnetic) energy is converted into particle kinetic energy. The energy production in the tube is related to the voltage difference $\left(\Phi_{\mathrm{e}}\right)$ between the footpoints of the loop. The dissipation rate is given by $L_{\text {diss }} \sim 10^{37} \Delta \phi\left(B / 10^{15} \mathrm{G}\right)\left(a / R_{\mathrm{NS}}\right)\left(e \Phi_{\mathrm{e}} / \mathrm{GeV}\right) \mathrm{erg} \mathrm{s}^{-1}$, where $\Delta \phi$ is the twist angle (in radians) and $a$ is the size of the twisted region. $e \Phi_{\mathrm{e}}$ must be sufficiently high for charged particles to be lifted from the surface of the neutron star $(\sim 200 \mathrm{MeV})$ or to create pairs in the magnetosphere $\left(\sim \gamma_{ \pm} m c^{2}=0.5 \gamma_{ \pm} \mathrm{MeV}\right)$ which are accelerated to $\sim 0.1-1 \mathrm{GeV}$.

If the voltage is too low to create a plasma, the tube regulates itself by either reducing the current or an electric field is generated $(\partial \boldsymbol{E} / \partial t \sim \nabla \times \boldsymbol{B}-\boldsymbol{j})$ until it is high enough to provide the plasma. If the voltage is too high, the parallel electric field will be screened by polarization of the plasma. Through this selfregulating voltage, "constant" pair production is achieved, feeding the high-energy emission. The dissipation time scale of the loop, i.e. the unwinding of the twist, is of the order 1-10 years. It is determined by the voltage difference $\Phi_{\mathrm{e}}$ and the corona luminosity $L_{\text {diss }}$. For a global twist of a dipole this becomes: $t_{\text {decay }}=0.8\left(R_{\mathrm{NS}} / c^{2}\right) L_{\text {diss }} / \Phi_{\mathrm{e}}^{2}$. Therefore, the stronger a twist (brighter corona) the longer the life time.

The downward beam of electrons and positrons through the tube will heat a transition layer between the atmosphere and the corona up to $\sim 200 \mathrm{keV}$, radiating bremsstrahlung to cool. In this thermal transition layer additional pairs can be created after electron ion collisions. The emerging spectrum will have a power-law shape with index $\Gamma \sim 1$ below the break energy. These bremsstrahlung photons can convert into pairs if the photon energy exceeds $2 m_{\mathrm{e}} c^{2} / \sin \theta$. In that case synchrotron emission will 
be produced. Either way, the maximum energy of photons escaping the strong magnetic field is $\sim 1 \mathrm{MeV}$.

Beloborodov \& Thompson (2007) also address the production of non-thermal emission below the INTEGRAL-ISGRI energy window. In the $2-10 \mathrm{keV}$ band resonant upscattering of $\mathrm{keV}$ photons can explain the soft power-law like X-ray tail $(>5 \mathrm{keV})$ to the black-body component first described by Thompson et al. (2002) (see also Lyutikov \& Gavriil 2006; Güver et al. 2006; Fernández \& Thompson 2007). For the nonthermal optical and NIR emission four possible emission mechanisms are discussed with a curvature-radiation origin being the most plausible explanation. See also the interpretation of the NIR, optical and soft X-ray observations by Hulleman et al. (2004), Morii et al. (2005a) and Durant \& van Kerkwijk (2006c).

A critical aspect in the corona model is the dissipation time scale of the twist. This determines how long a quasi stable loop can produce a non-thermal component in the pulse profile. Once the loop vanishes, the pulse profile should drastically change shape. When a next starquake produces a new tube, a new nonthermal pulse at a different phase should appear in the profile. The unwinding of such quasi stable loops feeds the non-thermal emission, and it seems plausible also that long-term flux variability occurs. Our results give increasing evidence that the nonthermal emission and the global structure of the pulse profile are stable over many years. During the INTEGRAL observations covering more than three years, we do not see significant variation in intensity and spectral shape of the total and pulsed emission above $20 \mathrm{keV}$. Furthermore, the RXTE monitoring observations covering the eight years before the INTEGRAL observations give time-averaged spectra for the total and total pulsed emission, as well as for the spectrum of the main pulse (Ph III) in the pulse profile that connect smoothly to the INTEGRAL-ISGRI spectra. In addition, the pulse maxima and the minima in the pulse profiles measured by ISGRI and RXTE align, suggesting a very stable geometry and intensity at hard $\mathrm{X}$-rays over more than 10 years. This seems to be at the limit of what the corona model still can sustain for a bright corona.

The proposed bremsstrahlung spectrum with index $\Gamma \sim 1$ (Beloborodov \& Thompson 2007) is consistent with our total spectrum for $4 \mathrm{U} 0142+61$ with $\Gamma=0.93 \pm 0.06$ below the break energy. However, we showed that the total pulsed spectrum is much harder with $\Gamma=0.40 \pm 0.15$, and, as a consequence, the DC difference spectrum much softer, both not consistent with a bremsstrahlung origin. The DC spectrum extends at least up to $100 \mathrm{keV}$, given the pulsed fraction of $\sim 50 \%$ at these high energies.

Lyubarsky \& Eichler (2008) revisited the heating by the downward beam of electrons and positrons of the transition region and conclude that this mechanism is incapable of heating the atmosphere due to suppression of the required twostream instability. They propose, rather, that a hot, tenuous atmosphere forms with a temperature of $\sim 1-2 \mathrm{MeV}$, an order of magnitude larger than inferred by Thompson \& Beloborodov (2005) and Beloborodov \& Thompson (2007). In this hot atmosphere hard radiation is generated via bremsstrahlung emission. Furthermore, pairs are easily produced and fill the whole magnetosphere forming a hot corona. In the latter collisionless interaction of the primary beam with the pair plasma in the corona heats the pairs even more. Most of this coronal energy is released in the hard X-ray and soft gamma-ray bands by Comptonization, so that the overall spectrum of the source is a superposition of bremsstrahlung radiation from the hot atmosphere and Comptonization radiation from the extended corona. The latter radiates in all directions, and the spectrum extends to the $\mathrm{MeV}$ band. The Comptonization radiation dominates below $\sim 100 \mathrm{keV}$ with photon spectral slope of $\Gamma \sim 1-2$, with a bremsstrahlung spectrum $(\Gamma \sim 1)$ above this break energy. This overall spectral shape is not consistent with our results on $4 \mathrm{U} 0142+61$. The Comptonization spectral shape below the break energy might be marginally consistent with that of the total spectrum, but by far too soft for the total pulsed spectrum. Furthermore, a bremsstrahlung spectrum after the break energy would mean an upturn of the spectrum, extending up to the $\mathrm{MeV}$ band. This is inconsistent with the INTEGRAL-SPI results above $100 \mathrm{keV}$ and more importantly, with the COMPTEL upper limits in the $\mathrm{MeV}$ band. Finally, it is hard to imagine how a Comptonization component originating in an extended corona radiating in all directions can produce the phase distribution of 4U 0142+61 with well defined pulses and, for 4U 0142+61, a sharp minimum.

Our concerns on the long-term stability/variability of the corona model as expressed for the work by Beloborodov \& Thompson (2007), are also valid for the approach of Lyubarsky \& Eichler (2008).

\subsection{Compton upscattering model}

Baring \& Harding (2007) consider resonant, magnetic Compton upscattering to explain the hard X-ray emission. Ultrarelativistic electrons, accelerated along either open or closed magnetic field lines, upscatter the thermal soft X-ray atmospheric photons. This process is very effective close to the magnetar surface $\left(\lesssim 10 R_{\mathrm{NS}}\right)$ where the magnetic field is still strong. For high-B isolated pulsars it is shown that acceleration of electrons to ultra-relativistic energies can occur (e.g. Sturner 1995; Harding \& Muslimov 1998; Dyks \& Rudak 2000). However, if AXPs with their long periods also have a dipolar structure at the surface, the acceleration zone cap radii would become extremely small (on the order of $50 \mathrm{~m}$ ). As a result, the relativistic electrons would have to be concentrated in such a narrow column with charge densities far exceeding the Goldreich-Julian maximum density (Goldreich \& Julian 1969). They argue, however, that non-dipolar structure at the surface is energetically feasible, allowing the electron acceleration zone to cover a much larger range of colatitudes than assigned to a standard polar cap.

The Compton scattering formalism invokes full relativistic and relativistic QED effects in high magnetic fields. Referring to earlier studies by i.e. Daugherty \& Harding (1986); Ho \& Epstein (1989) and Gonthier et al. (2000), and applications in the magnetic Thomson limit for old gamma-ray burst scenarios (Dermer 1990; Baring 1994) and for pulsar contexts (Daugherty \& Harding 1989; Sturner et al. 1995), Baring \& Harding (2007) make a first attempt to calculate geometries and emission spectra produced in the "resonasphere" of AXPs for a simplified scenario. They consider the case for a magnetic dipole field geometry and an injected monoenergetic electron distribution as well as monoenergetic incident photons of energy commensurate with the thermal photon temperatures $k T \sim 0.5-1 \mathrm{keV}$ observed in AXPs. Important first conclusions are that the Compton resonasphere for long period AXPs is confined to within a few stellar radii of the surface at higher field locales, and that the emission spectra are considerably softer than the hard X-ray tails seen in AXPs, and extend to energies higher than allowed by the COMPTEL upper limits, as e.g. shown in Fig. 3. The spectra do break above a maximum photon energy which is dependent on the magnetic field and the Lorentz factor. Furthermore, the observed spectra depend very sensitively on the viewing direction of the observer with respect to the magnetospheric 
geometry. To investigate such predictions, phase-resolved spectroscopy as applied in this work offers a very sensitive diagnostic tool. However, more complicated modelling addressing nondipolar field topologies and more realistic electron and photon spectra are required before our detailed findings can be compared with the predictions for hard X-ray emission from resonant Compton upscattering in AXPs. What can be noted is that the preliminary example spectra derived by Baring \& Harding (2007) are too soft and extend to energies much higher than can be permitted by the COMPTEL upper limits.

\subsection{Concluding remark}

The above discussions indicate that our detailed results as summarized in the previous section require, for all attempts to model the non-thermal emission above $20 \mathrm{keV}$, consideration of a full three-dimensional geometry taking the relevant angles (magnetic axis with respect to spin axis, viewing angle) into account, as well as the physical production processes taking place on the surface of the neutron star and in different sites of the atmosphere and magnetosphere. Such an analysis would be similar to what is done to model the non-thermal emission from radio pulsars. Our results seem to indicate that the non-thermal emission from AXPs is persistent, requiring a scenario with a stable geometry. To obtain further information on the geometry, including more information for the different relevant angles, similar detailed results for the other non-thermally emitting AXPs (see Kuiper et al. 2006) are important to place further constraints on the theoretical modelling.

Acknowledgements. We acknowledge C. Winkler, INTEGRAL Project Scientist and the INTEGRAL team for the quick ToO response; F. Haberl for useful discussions on how to analyse the XMM-Newton timing data correctly. This work is supported by NWO, Netherlands Organisation for Scientific Research. R.D. is supported by the Natural Sciences and Engineering Research Council (NSERC) PGSD scholarship. F.P.G. is supported by the NASA Postdoctoral Program administered by Oak Ridge Associated Universities at NASA Goddard SpaceFlight Center. Additional support was provided by NSERC Discovery Grant Rgpin 228738-03, FQRNT, CIFAR, and CFI. VMK holds a Lorne Trottier Chair in Astrophysics \& Cosmology, a Canada Research Chair and is a R. Howard Webster Foundation Fellow of CIFAR. The results are based on observations with INTEGRAL, an ESA project with instruments and science data centre funded by ESA member states (especially the PI countries: Denmark, France, Germany, Italy, Switzerland, Spain), Czech Republic and Poland, and with the participation of Russia and the USA. The SPI project has been completed under the responsibility and leadership of CNES. We are grateful to ASI, CEA, CNES, DLR, ESA, INTA, NASA and OSTC for support. This research has made use of data obtained through the High-Energy Astrophysics Center Online Service, provided by the NASA/Goddard Space-Flight Center.

\section{References}

Arnaud, K. A. 1996, in Astronomical Data Analysis Software and Systems V, ASP Conf. Ser., 101, 17

Baring, M. G. 1994, in American Institute of Physics Conf. Ser., Gamma-Ray Bursts, ed. G. J. Fishman, 307, 572

Baring, M. G., \& Harding, A. K. 2007, Ap\&SS, 308, 109

Beloborodov, A. M., \& Thompson, C. 2007, ApJ, 657, 967

Buccheri, R., Bennett, K., Bignami, G. F., et al. 1983, A\&A, 128, 245

Cheng, K. S., \& Zhang, L. 2001, ApJ, 562, 918

Daugherty, J. K., \& Harding, A. K. 1986, ApJ, 309, 362

Daugherty, J. K., \& Harding, A. K. 1989, ApJ, 336, 861

den Hartog, P. R., Kuiper, L., Hermsen, W., \& Vink, J. 2004, The Astronomer's Telegram, 293, 1

den Hartog, P. R., Hermsen, W., Kuiper, L., et al. 2006, A\&A, 451, 587

den Hartog, P. R., Kuiper, L., Hermsen, W., et al. 2007, Ap\&SS, 308, 647

Dermer, C. D. 1990, ApJ, 360, 197

Dhillon, V. S., Marsh, T. R., Hulleman, F., et al. 2005, MNRAS, 363, 609

Dib, R., Kaspi, V., Gavriil, F., \& Woods, P. 2006, The Astronomer's Telegram, 845,1

Dib, R., Kaspi, V. M., \& Gavriil, F. P. 2007, ApJ, 666, 1152
Duncan, R. C., \& Thompson, C. 1992, ApJ, 392, L9

Durant, M., \& van Kerkwijk, M. H. 2006a, ApJ, 650, 1070

Durant, M., \& van Kerkwijk, M. H. 2006b, ApJ, 650, 1082

Durant, M., \& van Kerkwijk, M. H. 2006c, ApJ, 652, 576

Dyks, J., \& Rudak, B. 2000, A\&A, 360, 263

Eadie, W. T., Drijard, D., \& James, F. E. 1971, Statistical methods in experimental physics (Amsterdam, North-Holland)

Eichler, D., Gedalin, M., \& Lyubarsky, Y. 2002, ApJ, 578, L121

Fernández, R., \& Thompson, C. 2007, ApJ, 660, 615

Gavriil, F. P., Dib, R., \& Kaspi, V. M. 2008, in 40 Years of Pulsars: Millisecond Pulsars, Magnetars and More, ed. C.-G. Bassa, Z. Wang, A. Cumming, \& V. M. Kaspi, AIP Conf. Ser., 983, 234

Gavriil, F. P., Dib, R., Kaspi, V. M., \& Woods, P. M. 2007, The Astronomer's Telegram, 993, 1

Gavriil, F. P., \& Kaspi, V. M. 2002, ApJ, 567, 1067

Goldreich, P., \& Julian, W. H. 1969, ApJ, 157, 869

Goldwurm, A., David, P., Foschini, L., et al. 2003, A\&A, 411, L223

Gonthier, P. L., Harding, A. K., Baring, M. G., Costello, R. M., \& Mercer, C. L. 2000, ApJ, 540, 907

Gonzalez, M. E., Dib, R., Kaspi, V. M., et al. 2008, ApJ, submitted

[arXiv: 0708.2756]

Güver, T., Özel, F., \& Göğüss, E. 2008, ApJ, 675, 1499

Güver, T., Özel, F., \& Lyutikov, M. 2006, ApJ, submitted [arXiv: astro-ph/0611405]

Harding, A. K., \& Muslimov, A. G. 1998, ApJ, 508, 328

Heyl, J. S. 2007, Ap\&SS, 308, 101

Heyl, J. S., \& Hernquist, L. 2005a, ApJ, 618, 463

Heyl, J. S., \& Hernquist, L. 2005b, MNRAS, 362, 777

Ho, C., \& Epstein, R. I. 1989, ApJ, 343, 277

Hulleman, F., van Kerkwijk, M. H., \& Kulkarni, S. R. 2004, A\&A, 416, 1037

Israel, G. L., Oosterbroek, T., Angelini, L., et al. 1999, A\&A, 346, 929

Jahoda, K., Swank, J. H., Giles, A. B., et al. 1996, in EUV, X-Ray, and Gamma-Ray Instrumentation for Astronomy VII, ed. O. H. Siegmund, \& M. A. Gummin, Proc. SPIE, 2808, 59

Jansen, F., Lumb, D., Altieri, B., et al. 2001, A\&A, 365, L1

Jensen, P. L., Clausen, K., Cassi, C., et al. 2003, A\&A, 411, L7

Juett, A. M., Marshall, H. L., Chakrabarty, D., \& Schulz, N. S. 2002, ApJ, 568, L31

Kaspi, V., Dib, R., \& Gavriil, F. 2006, The Astronomer's Telegram, 794, 1

Kaspi, V. M. 2007, Ap\&SS, 308, 1

Kern, B., \& Martin, C. 2002, Nature, 417, 527

Knödlseder, J. 2004, in 5th INTEGRAL Workshop on the INTEGRAL Universe, ed. V. Schoenfelder, G. Lichti, \& C. Winkler, ESA SP, 552, 867

Kuiper, L., Hermsen, W., \& Mendez, M. 2004, ApJ, 613, 1173

Kuiper, L., Hermsen, W., den Hartog, P. R., \& Collmar, W. 2006, ApJ, 645, 556

Lebrun, F., Leray, J. P., Lavocat, P., et al. 2003, A\&A, 411, L141

Lyubarsky, Y., \& Eichler, D. 2008, ApJ, submitted [arXiv : 0706. 3578]

Lyutikov, M., \& Gavriil, F. P. 2006, MNRAS, 368, 690

Makishima, K., Tashiro, M., Ebisawa, K., et al. 1996, PASJ, 48, 171

Molkov, S. V., Cherepashchuk, A. M., Lutovinov, A. A., et al. 2004, Astron. Lett., 30, 534

Morii, M., Kawai, N., Kataoka, J., et al. 2005a, Adv. Space Res., 35, 1177

Morii, M., Kawai, N., \& Shibazaki, N. 2005b, ApJ, 622, 544

Motch, C., Belloni, T., Buckley, D., et al. 1991, A\&A, 246, L24

Ohashi, T., Ebisawa, K., Fukazawa, Y., et al. 1996, PASJ, 48, 157

Rea, N., Nichelli, E., Israel, G. L., et al. 2007a, MNRAS, 381, 293

Rea, N., Turolla, R., Zane, S., et al. 2007b, ApJ, 661, L65

Revnivtsev, M. G., Sunyaev, R. A., Varshalovich, D. A., et al. 2004, Astron. Lett., 30, 382

Strüder, L., Briel, U., Dennerl, K., et al. 2001, A\&A, 365, L18

Sturner, S. J. 1995, ApJ, 446, 292

Sturner, S. J., Dermer, C. D., \& Michel, F. C. 1995, ApJ, 445, 736

Tanaka, Y., Inoue, H., \& Holt, S. S. 1994, PASJ, 46, L37

Thompson, C., \& Duncan, R. C. 1993, ApJ, 408, 194

Thompson, C., \& Duncan, R. C. 1995, MNRAS, 275, 255

Thompson, C., \& Duncan, R. C. 1996, ApJ, 473, 322

Thompson, C., \& Beloborodov, A. M. 2005, ApJ, 634, 565

Thompson, C., Duncan, R. C., Woods, P. M., et al. 2000, ApJ, 543, 340

Thompson, C., Lyutikov, M., \& Kulkarni, S. R. 2002, ApJ, 574, 332

Turner, M. J. L., Abbey, A., Arnaud, M., et al. 2001, A\&A, 365, L27

Ubertini, P., Lebrun, F., Di Cocco, G., et al. 2003, A\&A, 411, L131

Valinia, A., \& Marshall, F. E. 1998, ApJ, 505, 134

Vedrenne, G., Roques, J.-P., Schönfelder, V., et al. 2003, A\&A, 411, L63

Walter, R., Favre, P., Dubath, P., et al. 2003, A\&A, 411, L25

Winkler, C., Courvoisier, T. J.-L., Di Cocco, G., et al. 2003, A\&A, 411, L1

Woods, P. M., \& Thompson, C. 2006, in Compact stellar X-ray sources, ed. W. Lewin, \& M. van der Klis, Cambridge Astrophys. Ser., 39, 547 